\title{
Pareto Optimality Conditions and Duality for Vector Quadratic Fractional Optimization Problems
}

\author{
W. A. Oliveira, ${ }^{1}$ A. Beato-Moreno, ${ }^{2}$ A. C. Moretti, ${ }^{1}$ and L. L. Salles Neto ${ }^{3}$ \\ ${ }^{1}$ School of Applied Sciences, State University of Campinas, 13484-350 Limeira, SP, Brazil \\ ${ }^{2}$ Department of Statistics and Operations Research, College of Mathematics, University of Sevilla, 41012 Sevilla, Spain \\ ${ }^{3}$ Department of Science and Technology, Federal University of São Paulo, 12247-014 São José dos Campos, SP, Brazil
}

Correspondence should be addressed to W. A. Oliveira; washington.oliveira@fca.unicamp.br

Received 12 March 2014; Revised 25 July 2014; Accepted 6 August 2014; Published 25 November 2014

Academic Editor: Chao Yan

Copyright (C) 2014 W. A. Oliveira et al. This is an open access article distributed under the Creative Commons Attribution License, which permits unrestricted use, distribution, and reproduction in any medium, provided the original work is properly cited.

\begin{abstract}
One of the most important optimality conditions to aid in solving a vector optimization problem is the first-order necessary optimality condition that generalizes the Karush-Kuhn-Tucker condition. However, to obtain the sufficient optimality conditions, it is necessary to impose additional assumptions on the objective functions and on the constraint set. The present work is concerned with the constrained vector quadratic fractional optimization problem. It shows that sufficient Pareto optimality conditions and the main duality theorems can be established without the assumption of generalized convexity in the objective functions, by considering some assumptions on a linear combination of Hessian matrices instead. The main aspect of this contribution is the development of Pareto optimality conditions based on a similar second-order sufficient condition for problems with convex constraints, without convexity assumptions on the objective functions. These conditions might be useful to determine termination criteria in the development of algorithms.
\end{abstract}

\section{Introduction}

There are many contributions, concepts, and definitions that characterize and give the Pareto optimality conditions for solutions of a vector optimization problem (see, for instance, $[1,2])$. One of the most important condition is the firstorder necessary optimality condition that generalizes the Karush-Kuhn-Tucker (KKT) condition. However, to obtain the sufficient optimality conditions, it is necessary to impose additional assumptions (like convexity and its generalizations) on the objective functions and on the constraint set.

In this paper, we deal with a particular case of vector optimization problem (VOP), where each objective function consists of a ratio of two quadratic functions. Without generalized convexity assumptions on the objective functions, but by imposing some additional assumptions on a linear combination of Hessian matrices, Pareto optimality conditions are obtained and duality theorems are established.
Let us consider the following vector quadratic fractional optimization problem (VQFP):

$$
\text { Minimize } \frac{f(x)}{g(x)}=\left(\frac{f_{1}(x)}{g_{1}(x)}, \ldots, \frac{f_{m}(x)}{g_{m}(x)}\right)
$$

subject to $h_{j}(x) \leqq 0 \quad j \in J, x \in \Omega$,

where $\Omega \subseteq \mathbb{R}^{n}$ is an open set, $f_{i}, g_{i}, i \in I \equiv\{1, \ldots, m\}$, and $h_{j}, j \in J \equiv\{1, \ldots, \ell\}$, are continuously differentiable realvalued functions defined on $\Omega$. In addition, we assume that $f_{i}, g_{i}, i \in I$, are quadratic functions and $g_{i}(x)>0$ for $x \in \Omega$ and $i \in I$. We denote by $S$ the feasible set of elements $x \in \Omega$ satisfying $h_{j}(x) \leqq 0$. We say that $x$ is a feasible point if $x \in S$. The value $f_{i}(x) / g_{i}(x)$ is the result of the $i$ th objective function if the decision maker chooses the action $x \in S$. 
Fractional optimization problems arise frequently in decision making applications, including science management, portfolio selection, cutting and stock, and game theory, in the optimization of the ratio performance/cost, or profit/investment, or cost/time.

There are many contributions dealing with the scalar (single-objective) fractional optimization problem (FP) and vector fractional optimization problem (VFP). In most of them, using convexity or generalized convexity, optimality conditions in the KKT sense and the main duality theorems for optimal points are obtained. With a parametric approach, which transforms the original problem in a simpler associated problem, Dinkelbach [3], Jagannathan [4], and Antczak [5] established optimality conditions, presented algorithms, and applied their approaches in an example (FP) consisting of quadratic functions. Using some known generalized convexity, Antczak [5], Khan and Hanson [6], Reddy and Mukherjee [7], Jeyakumar [8], and Liang et al. [9] established optimality conditions and theorems that relate the pair primal-dual of problem (FP). In Craven [10] and Weir [11], other results for the scalar optimization (FP) can be found.

Further, Liang et al. [12] extended their approach to the vector optimization case (VFP) considering the type duals of Mond and Weir [13], Schaible [14], and Bector [15]. Considering the parametric approach of Dinkelbach [3], Jagannathan [4], and Bector et al. [16] and two classes of generalized convexity, Osuna-Gómez et al. [17] established weak Pareto optimality conditions and the main duality theorems for the differentiable vector optimization case (VFP). Santos et al. [18] deepened these results to the more general nondifferentiable case (VFP). Jeyakumar and Mond [19] used generalized convexity to study the problem (VFP).

Few studies are found involving quadratic functions at both the numerator and denominator of the ratio objective function. Most of them involve the mixing of linear and quadratic functions. The most similar approaches to the scalar quadratic fractional optimization problem (QFP) were considered in [20-24]. On the other hand, Benson [25] considered a pure (QFP) consisting of convex functions, where some theoretical properties and optimality conditions are developed, and an algorithm and its convergence properties are presented.

The closest approaches to the vector optimization case (VQFP) were considered in [26-33]. Using an iterative computational test, Beato et al. [27, 28] characterized the Pareto optimal point for the problem (VQFP), consisting of linear and quadratic functions, and some theoretical results were obtained by using the function linearization technique of Bector et al. [16]. Arévalo and Zapata [26], Konno and Inori [29], and Rhode and Weber [33] analyzed the portfolio selection problem. Kornbluth and Steuer [32] used an adapted Simplex method in the problem (VFP) consisting of linear functions. Korhonen and $\mathrm{Yu}[30,31]$ proposed an iterative computational method for solving the problem (VQFP), consisting of linear and quadratic functions, based on search directions and weighted sums.

The approach taken in this work is different from the previous ones. The main aspect of this contribution is the development of Pareto optimality conditions for a particular vector optimization problem based on a similar second-order sufficient condition for Pareto optimality for problems with convex constraints without the hypothesis of convexity on the objective functions. These conditions might be useful to determine termination criteria in the development of algorithms, and new extensions can be established from these, where more general vector optimization problems in which algorithms are based on quadratic approximations are used locally.

This paper is organized as follows. We start by defining some notations and basic properties in Section 2. In Section 3, the sufficient Pareto optimality conditions are established. In Section 4, the relationship among the associated problems is presented and duality theorems are established. Finally, comments and concluding remarks are presented in Section 5.

\section{Preliminaries}

Let $\mathbb{R}_{+}$denote the nonnegative real numbers and let $x^{T}$ denote the transpose of the vector $x \in \mathbb{R}^{n}$. Furthermore, we will adopt the following conventions for inequalities among vectors. If $x=\left(x_{1}, \ldots, x_{m}\right)^{T} \in \mathbb{R}^{m}$ and $y=\left(y_{1}, \ldots, y_{m}\right)^{T} \in$ $\mathbb{R}^{m}$, then

$$
\begin{aligned}
& x=y \text { if and only if } x_{i}=y_{i}, \forall i \in I \\
& x<y \text { if and only if } x_{i}<y_{i}, \forall i \in I \\
& x \leqq y \text { if and only if } x_{i} \leqq y_{i}, \forall i \in I \\
& x \leq y \text { if and only if } x \leqq y \text { and } x \neq y .
\end{aligned}
$$

Similarly we consider the equivalent convention for inequalities $>$, $\geqq$, and $\geq$.

Different optimality definitions for the problem (VQFP) are referred to as Pareto optimal solutions [34], two of which are defined as follows.

Definition 1. Feasible point $x^{*}$ is said to be a Pareto optimal solution of (VQFP), if there does not exist another $x \in S$ such that $f(x) / g(x) \leq f\left(x^{*}\right) / g\left(x^{*}\right)$.

Definition 2. Feasible point $x^{*}$ is said to be a weakly Pareto optimal solution of (VQFP), if there does not exist another $x \in S$ such that $f(x) / g(x)<f\left(x^{*}\right) / g\left(x^{*}\right)$.

Hypotheses of convexity or generalized convexity on the objective functions will be avoided in this work, but we will use such hypotheses on the constraint set. We recall the definition of convexity, where $\nabla f(x)$ denotes the gradient of the function $f: \mathbb{R}^{n} \rightarrow \mathbb{R}$ at the point $x$.

Definition 3. Let $f: \Omega \subseteq \mathbb{R}^{n} \rightarrow \mathbb{R}$ be a function defined on an open convex set $\Omega$ and differentiable at $x^{*} \in \Omega$. $f$ is called convex at $x^{*}$ if, for all $x \in \Omega, f(x)-f\left(x^{*}\right) \geqq \nabla f\left(x^{*}\right)^{T}\left(x-x^{*}\right)$. When $f$ is convex on the set $\Omega$, we simply say that $f$ is convex.

Maeda [35] used the generalized Guignard constraint qualification (GGCQ) [36], to derive the following necessary Pareto optimality conditions for the problem (VOP) in the KKT sense. Assuming differentiability of the objective and the constraint functions, Maeda guarantees the existence of 
Lagrange multipliers, all strictly positive, associated with the objective functions.

Lemma 4 (Maeda [35]). Let $x^{*}$ be a Pareto optimal solution of (VQFP). Suppose that (GGCQ) holds at $x^{*}$; then there exist vectors $\tau \in \mathbb{R}^{m}, \lambda \in \mathbb{R}^{\ell}$ such that

$$
\begin{array}{r}
\sum_{i=1}^{m} \tau_{i} \nabla \frac{f_{i}\left(x^{*}\right)}{g_{i}\left(x^{*}\right)}+\sum_{j=1}^{\ell} \lambda_{j} \nabla h_{j}\left(x^{*}\right)=0, \\
\sum_{j=1}^{\ell} \lambda_{j} h_{j}\left(x^{*}\right)=0, \\
\tau>0, \quad \lambda \geqq 0 .
\end{array}
$$

For each $i \in I$ and $x \in \mathbb{R}^{n}$, we consider the objective functions defined as $f_{i}(x)=x^{T} A_{i} x+a_{i}^{T} x+\bar{a}_{i}$ and $g_{i}(x)=$ $x^{T} B_{i} x+b_{i}^{T} x+\bar{b}_{i}$, where $A_{i}, B_{i} \in \mathbb{R}^{n \times n}, A_{i}$ is symmetric, $B_{i}$ is symmetric and positive semidefinite, and $a_{i}, b_{i} \in \mathbb{R}^{n}$ and $\bar{a}_{i}$, $\bar{b}_{i} \in \mathbb{R}$, with $\bar{b}_{i}>-\left(w^{i^{T}} B_{i} w^{i}+b_{i}^{T} w^{i}\right)$, where $w^{i}$ is the solution of the system $2 B_{i} x+b_{i}=0$; that is, $w^{i}$ is the point in which the function $x^{T} B_{i} x+b_{i}^{T} x$ reaches its minimum and this ensures that $g_{i}(x)>0, \forall x \in \mathbb{R}^{n}$. We cannot consider the cases where $2 B_{i} x+b_{i}=0$ has no solution.

\section{Sufficient Optimality Conditions}

Without assumptions of generalized convexity, but imposing some additional assumptions on a linear combination of Hessian matrices of the objective functions $f_{i}$ and $g_{i}, i \in$ $I$, we provide in the next theorem a sufficient condition that guarantees that a feasible point of (VQFP) is Pareto optimal point. Similar to a second-order sufficient condition for Pareto optimality, this condition explores the intrinsic characteristics of the problem (VQFP).

We assume, unlike the objective functions, that each $h_{j}$ is convex. Also, given $x^{*} \in S$, for each $i \in I$, we define the scalar functions $u_{i}: S \times S \rightarrow \mathbb{R}_{+} \backslash\{0\}$ and $s_{i}: S \times S \rightarrow \mathbb{R}$ by

$$
\begin{aligned}
& u_{i}\left(x, x^{*}\right) \\
& \quad \equiv \frac{g_{i}\left(x^{*}\right)}{g_{i}(x)}, \\
& s_{i}\left(x, x^{*}\right) \\
& \quad \equiv \frac{1}{g_{i}(x)}\left\{\left(x-x^{*}\right)^{T}\left[A_{i}-\frac{f_{i}\left(x^{*}\right)}{g_{i}\left(x^{*}\right)} B_{i}\right]\left(x-x^{*}\right)\right\} .
\end{aligned}
$$

Theorem 5. Let $x^{*}$ be a feasible point of (VQFP). Suppose that the constraint function $h_{j}$ is convex for each $j \in J$ and there exist vectors $\tau \in \mathbb{R}^{m}, \lambda \in \mathbb{R}^{\ell}$, such that

$$
\begin{array}{r}
\sum_{i=1}^{m} \tau_{i} \nabla \frac{f_{i}\left(x^{*}\right)}{g_{i}\left(x^{*}\right)}+\sum_{j=1}^{\ell} \lambda_{j} \nabla h_{j}\left(x^{*}\right)=0, \\
\sum_{j=1}^{\ell} \lambda_{j} h_{j}\left(x^{*}\right)=0, \\
\tau>0, \quad \lambda \geqq 0 .
\end{array}
$$

If, for any $x \in S$, we obtain

$$
\sum_{i=1}^{m} \tau_{i} \frac{s_{i}\left(x, x^{*}\right)}{u_{i}\left(x, x^{*}\right)} \geqq 0,
$$

then $x^{*}$ is a Pareto optimal solution for (VQFP).

Proof. Given $x \in S$, we obtain for each $i \in I$

$$
\begin{aligned}
& f_{i}(x)-f_{i}\left(x^{*}\right) \\
& =\left(x-x^{*}\right)^{T} A_{i}\left(x-x^{*}\right)+\nabla f_{i}\left(x^{*}\right)^{T}\left(x-x^{*}\right), \\
& g_{i}(x)-g_{i}\left(x^{*}\right) \\
& =\left(x-x^{*}\right)^{T} B_{i}\left(x-x^{*}\right)+\nabla g_{i}\left(x^{*}\right)^{T}\left(x-x^{*}\right), \\
& \frac{f_{i}(x)}{g_{i}(x)}-\frac{f_{i}\left(x^{*}\right)}{g_{i}\left(x^{*}\right)} \\
& =\frac{f_{i}(x) g_{i}\left(x^{*}\right)-g_{i}(x) f_{i}\left(x^{*}\right)}{g_{i}(x) g_{i}\left(x^{*}\right)} \\
& =\left(f_{i}(x) g_{i}\left(x^{*}\right)-f_{i}\left(x^{*}\right) g_{i}\left(x^{*}\right)+f_{i}\left(x^{*}\right) g_{i}\left(x^{*}\right)\right. \\
& \left.-g_{i}(x) f_{i}\left(x^{*}\right)\right) \\
& \cdot\left(g_{i}(x) g_{i}\left(x^{*}\right)\right)^{-1} \\
& =\frac{g_{i}\left(x^{*}\right)\left\{f_{i}(x)-f_{i}\left(x^{*}\right)\right\}-f_{i}\left(x^{*}\right)\left\{g_{i}(x)-g_{i}\left(x^{*}\right)\right\}}{g_{i}(x) g_{i}\left(x^{*}\right)} \\
& =\frac{1}{g_{i}(x)}\left\{\left(x-x^{*}\right)^{T} A_{i}\left(x-x^{*}\right)+\nabla f_{i}\left(x^{*}\right)^{T}\left(x-x^{*}\right)\right\} \\
& -\frac{f_{i}\left(x^{*}\right)}{g_{i}(x) g_{i}\left(x^{*}\right)} \\
& \cdot\left\{\left(x-x^{*}\right)^{T} B_{i}\left(x-x^{*}\right)+\nabla g_{i}\left(x^{*}\right)^{T}\left(x-x^{*}\right)\right\} \\
& =\frac{1}{g_{i}(x)}\left\{\left(x-x^{*}\right)^{T} A_{i}\left(x-x^{*}\right)\right\} \\
& -\frac{f_{i}\left(x^{*}\right)}{g_{i}(x) g_{i}\left(x^{*}\right)}\left\{\left(x-x^{*}\right)^{T} B_{i}\left(x-x^{*}\right)\right\} \\
& +\frac{1}{g_{i}(x)}\left\{\nabla f_{i}\left(x^{*}\right)^{T}\left(x-x^{*}\right)\right\} \\
& -\frac{f_{i}\left(x^{*}\right)}{g_{i}(x) g_{i}\left(x^{*}\right)}\left\{\nabla g_{i}\left(x^{*}\right)^{T}\left(x-x^{*}\right)\right\} \\
& =\frac{1}{g_{i}(x)}\left\{\left(x-x^{*}\right)^{T}\left[A_{i}-\frac{f_{i}\left(x^{*}\right)}{g_{i}\left(x^{*}\right)} B_{i}\right]\left(x-x^{*}\right)\right\} \\
& +\frac{g_{i}\left(x^{*}\right)}{g_{i}(x)} \\
& \left\{\left[\frac{\nabla f_{i}\left(x^{*}\right) g_{i}\left(x^{*}\right)-\nabla g_{i}\left(x^{*}\right) f_{i}\left(x^{*}\right)}{\left[g_{i}\left(x^{*}\right)\right]^{2}}\right]^{T}\left(x-x^{*}\right)\right\}
\end{aligned}
$$




$$
\begin{aligned}
= & \frac{1}{g_{i}(x)}\left\{\left(x-x^{*}\right)^{T}\left[A_{i}-\frac{f_{i}\left(x^{*}\right)}{g_{i}\left(x^{*}\right)} B_{i}\right]\left(x-x^{*}\right)\right\} \\
& +\frac{g_{i}\left(x^{*}\right)}{g_{i}(x)}\left\{\left[\nabla \frac{f_{i}\left(x^{*}\right)}{g_{i}\left(x^{*}\right)}\right]^{T}\left(x-x^{*}\right)\right\} .
\end{aligned}
$$

Thus, each function $f_{i} / g_{i}$ satisfies

$$
\begin{aligned}
\frac{f_{i}(x)}{g_{i}(x)}-\frac{f_{i}\left(x^{*}\right)}{g_{i}\left(x^{*}\right)}= & u_{i}\left(x, x^{*}\right)\left[\nabla \frac{f_{i}\left(x^{*}\right)}{g_{i}\left(x^{*}\right)}\right]^{T}\left(x-x^{*}\right) \\
& +s_{i}\left(x, x^{*}\right), \quad \forall x \in S .
\end{aligned}
$$

Suppose that $x^{*}$ is not a Pareto optimal solution of (VQFP). Then there exists another point $x \in S$ such that

$$
\frac{f(x)}{g(x)} \leq \frac{f\left(x^{*}\right)}{g\left(x^{*}\right)} .
$$

Since $u_{i}\left(x, x^{*}\right)>0, i \in I$, from (8) we obtain

$$
\begin{aligned}
& \frac{1}{u_{i}\left(x, x^{*}\right)}\left(\frac{f_{i}(x)}{g_{i}(x)}-\frac{f_{i}\left(x^{*}\right)}{g_{i}\left(x^{*}\right)}\right) \\
& =\left[\nabla \frac{f_{i}\left(x^{*}\right)}{g_{i}\left(x^{*}\right)}\right]^{T}\left(x-x^{*}\right)+\frac{s_{i}\left(x, x^{*}\right)}{u_{i}\left(x, x^{*}\right)}, \quad i \in I .
\end{aligned}
$$

From (9), we have

$$
\frac{f(x)}{g(x)}-\frac{f\left(x^{*}\right)}{g\left(x^{*}\right)} \leq 0,
$$

and we obtain $m$ inequalities

$$
\left[\nabla \frac{f_{i}\left(x^{*}\right)}{g_{i}\left(x^{*}\right)}\right]^{T}\left(x-x^{*}\right)+\frac{s_{i}\left(x, x^{*}\right)}{u_{i}\left(x, x^{*}\right)} \leqq 0, \quad i \in I,
$$

with at least one strict inequality. Multiplying the $m$ inequalities above by their respective $\tau_{i}>0, i \in I$, and summing all the products, we obtain

$$
\sum_{i=1}^{m} \tau_{i}\left[\nabla \frac{f_{i}\left(x^{*}\right)}{g_{i}\left(x^{*}\right)}\right]^{T}\left(x-x^{*}\right)+\sum_{i=1}^{m} \tau_{i} \frac{s_{i}\left(x, x^{*}\right)}{u_{i}\left(x, x^{*}\right)}<0 .
$$

Then, we have

$$
\left[\sum_{i=1}^{m} \tau_{i} \nabla \frac{f_{i}\left(x^{*}\right)}{g_{i}\left(x^{*}\right)}\right]^{T}\left(x-x^{*}\right)+\sum_{i=1}^{m} \tau_{i} \frac{s_{i}\left(x, x^{*}\right)}{u_{i}\left(x, x^{*}\right)}<0 .
$$

Substituting (3) into (14), we get

$$
\left[-\sum_{j=1}^{\ell} \lambda_{j} \nabla h_{j}\left(x^{*}\right)\right]^{T}\left(x-x^{*}\right)+\sum_{i=1}^{m} \tau_{i} \frac{s_{i}\left(x, x^{*}\right)}{u_{i}\left(x, x^{*}\right)}<0 .
$$

Using (6) and (15), we obtain

$$
0 \leqq \sum_{i=1}^{m} \tau_{i} \frac{s_{i}\left(x, x^{*}\right)}{u_{i}\left(x, x^{*}\right)}<\left[\sum_{j=1}^{\ell} \lambda_{j} \nabla h_{j}\left(x^{*}\right)\right]^{T}\left(x-x^{*}\right) .
$$

That is,

$$
\left[\sum_{j=1}^{\ell} \lambda_{j} \nabla h_{j}\left(x^{*}\right)\right]^{T}\left(x-x^{*}\right)>0 .
$$

On the other hand, by convexity of $h_{j}$, we have, for each $j \in J$,

$$
h_{j}(x)-h_{j}\left(x^{*}\right) \geqq \nabla h_{j}\left(x^{*}\right)^{T}\left(x-x^{*}\right) .
$$

Since $\lambda_{j} \geqq 0, j \in J$, we have

$$
\begin{aligned}
\sum_{j=1}^{\ell} \lambda_{j}\left(h_{j}(x)-h_{j}\left(x^{*}\right)\right) & \geqq \sum_{j=1}^{\ell} \lambda_{j} \nabla h_{j}\left(x^{*}\right)^{T}\left(x-x^{*}\right) \\
& =\left[\sum_{j=1}^{\ell} \lambda_{j} \nabla h_{j}\left(x^{*}\right)\right]^{T}\left(x-x^{*}\right) .
\end{aligned}
$$

However, since $x$ is feasible point, condition (4) and $\lambda_{j} \geqq 0$, $j \in J$, imply that

$$
\sum_{j=1}^{\ell} \lambda_{j}\left(h_{j}(x)-h_{j}\left(x^{*}\right)\right) \leqq 0 .
$$

We conclude that

$$
\left[\sum_{j=1}^{\ell} \lambda_{j} \nabla h_{j}\left(x^{*}\right)\right]^{T}\left(x-x^{*}\right) \leqq 0,
$$

which contradicts (17). Therefore $x^{*}$ is a Pareto optimal solution for (VQFP).

The expression $f_{i}(x) / g_{i}(x)-f_{i}\left(x^{*}\right) / g_{i}\left(x^{*}\right)$ in Theorem 5 is manipulated in a similar manner in $[6,7,9,12,19]$; however some generalized convexity on the functions $f_{i}$ and $g_{i}$ is imposed. In most of them, for each $i \in I$ and $x \in S$, the hypotheses $f_{i}(x) \geqq 0, g_{i}(x)>0$, and $f_{i},-g_{i}$ satisfy some generalized convexity. This is not the purpose of this work, but the constraint functions can be assumed in a more general class of convex functions; for example, the generalized convexity of Liang et al. [9] can be used.

In the following, the Pareto optimal solution set is denoted by Eff (VQFP).

Corollary 6. Let $x^{*}$ be a feasible point of (VQFP). Suppose that the constraint function $h_{j}$ is convex for each $j \in J$ and there exist vectors $\tau \in \mathbb{R}^{m}, \lambda \in \mathbb{R}^{\ell}$, such that (3), (4), and (5) are valid. If $\left[A_{i}-\left(f_{i}\left(x^{*}\right) / g_{i}\left(x^{*}\right)\right) B_{i}\right]$ are positive semidefinite matrices for each $i \in I$, then $x^{*} \in \mathrm{Eff}(V Q F P)$. 
Proof. By hypothesis, given $x \in S$ and $i \in I$, we obtain

$$
\begin{aligned}
& \left(x-x^{*}\right)^{T}\left[A_{i}-\frac{f_{i}\left(x^{*}\right)}{g_{i}\left(x^{*}\right)} B_{i}\right]\left(x-x^{*}\right) \geqq 0 \\
& \Longrightarrow \tau_{i} \frac{1}{g_{i}\left(x^{*}\right)}\left\{\left(x-x^{*}\right)^{T}\left[A_{i}-\frac{f_{i}\left(x^{*}\right)}{g_{i}\left(x^{*}\right)} B_{i}\right]\left(x-x^{*}\right)\right\} \geqq 0 \\
& \Longrightarrow \sum_{i=1}^{m} \tau_{i} \frac{g_{i}(x)}{g_{i}\left(x^{*}\right)} \frac{1}{g_{i}(x)} \\
& \quad \cdot\left\{\left(x-x^{*}\right)^{T}\left[A_{i}-\frac{f_{i}\left(x^{*}\right)}{g_{i}\left(x^{*}\right)} B_{i}\right]\left(x-x^{*}\right)\right\} \geqq 0 \\
& \Longrightarrow \sum_{i=1}^{m} \tau_{i} \frac{s_{i}\left(x, x^{*}\right)}{u_{i}\left(x, x^{*}\right)} \geqq 0 .
\end{aligned}
$$

Therefore, inequality (6) is valid and the result follows from Theorem 5 .

To ensure that inequality (6) is valid, we start exploring the features of the Hessian matrices of the objective functions of (VQFP).

Negative values can occur in each term $\tau_{i}\left(s_{i}\left(x, x^{*}\right) / u_{i}\left(x, x^{*}\right)\right)$ of the sum $\sum_{i=1}^{m} \tau_{i}\left(s_{i}\left(x, x^{*}\right) / u_{i}\left(x, x^{*}\right)\right)$, which depends on each matrix $\left[A_{i}-\left(f_{i}\left(x^{*}\right) / g_{i}\left(x^{*}\right)\right) B_{i}\right]$, $i \in I$, and the vector $\left(x-x^{*}\right)$. Let us check new conditions for which (6) is satisfied; that is, we want to ensure the result of Theorem 5 by analysing the function

$$
\begin{aligned}
& Z\left(x, x^{*}\right) \equiv \sum_{i=1}^{m} \tau_{i} \frac{s_{i}\left(x, x^{*}\right)}{u_{i}\left(x, x^{*}\right)} \\
& =\sum_{i=1}^{m} \tau_{i} \frac{g_{i}(x)}{g_{i}\left(x^{*}\right)} \frac{1}{g_{i}(x)} \\
& \quad \cdot\left\{\left(x-x^{*}\right)^{T}\left[A_{i}-\frac{f_{i}\left(x^{*}\right)}{g_{i}\left(x^{*}\right)} B_{i}\right]\left(x-x^{*}\right)\right\} \\
& =\left(x-x^{*}\right)^{T}\left\{\sum_{i=1}^{m}\left[\frac{\tau_{i}}{g_{i}\left(x^{*}\right)} A_{i}-\frac{\tau_{i} f_{i}\left(x^{*}\right)}{\left[g_{i}\left(x^{*}\right)\right]^{2}} B_{i}\right]\right\}\left(x-x^{*}\right) .
\end{aligned}
$$

Note that $Z\left(\cdot, x^{*}\right)$ is a quadratic function without the linear part; thus in (VQFP) we obtain $Z\left(x, x^{*}\right) \geqq 0$ on $S$ if and only if $\min _{x \in S} Z\left(x, x^{*}\right) \geqq 0$; that is, we can use the classical results on quadratic optimization to check if $\min _{x \in S} Z\left(x, x^{*}\right) \geqq 0$. The next corollary follows immediately from Theorem 5 .

Corollary 7. Let $x^{*}$ be a feasible point of (VQFP). Suppose that the constraint function $h_{j}$ is convex for each $j \in J$ and there exist vectors $\tau \in \mathbb{R}^{m}, \lambda \in \mathbb{R}^{\ell}$, such that (3), (4), and (5) are valid. If $\min _{x \in S} Z\left(x, x^{*}\right) \geqq 0$, then $x^{*} \in \mathrm{Eff}(V Q F P)$.
Using the previous results to check whether a feasible point is a Pareto optimal solution of (VQFP), we propose the following computational test method.

\section{Pareto Optimality Test}

Step 1. Given $x^{*} \in S$, find the vectors $\tau>0$ and $\lambda \geqq 0$ such that (3) and (4) are valid. If the vectors $\tau$ and $\lambda$ do not exist, then $x^{*} \notin \mathrm{Eff}(\mathrm{VQFP})$.

Step 2. Otherwise, solve $Z\left(\bar{x}, x^{*}\right)=\min _{x \in S} Z\left(x, x^{*}\right)$. If $Z\left(\bar{x}, x^{*}\right) \geqq 0$, we say that $x^{*}$ has passed the Pareto optimality test and $x^{*} \in \mathrm{Eff}(\mathrm{VQFP})$.

Pareto optimality test starts with a feasible point; then it seeks to solve a system of linear equations containing $m+\ell$ unknowns, $\tau$ and $\lambda$, the inequalities $\tau>0, \lambda \geqq 0$, and two equalities (3) and (4). If this system has no solution, then the point $x^{*}$ does not satisfy the first-order necessary condition for Pareto optimality, so the method terminates concluding that $x^{*} \notin \mathrm{Eff}(\mathrm{VQFP})$. Otherwise, in Step 2, a quadratic optimization problem on $S$ should be solved. If the minimum of the quadratic problem is nonnegative, then the procedure ends, concluding that $x^{*} \in \mathrm{Eff}(\mathrm{VQFP})$. Otherwise, we say that $x^{*}$ has not passed the Pareto optimality test. Its complexity lies in solving a system of linear inequalities plus a quadratic optimization problem.

The next results, which address a linear combination of the Hessian matrices, can be used to develop a computational search method.

Looking at the previous Pareto optimality test, if the fixed point $x^{*}$ is assumed to be a variable $y$, then the linear system in Step 1 becomes a nonlinear system for the variables $\tau>0$, $\lambda \geqq 0, y \in S$. And the quadratic optimization problem in Step 2 becomes a quadratic optimization problem of the type $\min _{x, y \in S} Z(x, y)$. This raises considerable difficulties. In order to reduce these difficulties, we further explore the characteristics of the matrix

$$
\widehat{F}\left(y^{*}\right) \equiv \sum_{i=1}^{m}\left[\frac{\tau_{i}}{g_{i}\left(y^{*}\right)} A_{i}-\frac{\tau_{i} f_{i}\left(y^{*}\right)}{\left[g_{i}\left(y^{*}\right)\right]^{2}} B_{i}\right] .
$$

One possibility is to search for points $y^{*}$ such that $\widehat{F}\left(y^{*}\right)$ becomes positive semidefinite. In this case $Z\left(x, y^{*}\right)=(x-$ $\left.y^{*}\right)^{T} \widehat{F}\left(y^{*}\right)\left(x-y^{*}\right) \geqq 0$ depends only on $y^{*} \in S$.

Consider a fixed point $x^{*}$; the next theorem takes advantage of the symmetry and diagonalizations of the matrices $A_{i}$ and $B_{i}, i \in I$, to give sufficient Pareto optimality conditions for a feasible point of (VQFP). Consider the usual inner product $\langle\cdot, \cdot\rangle$ in $\mathbb{R}^{n}$.

Theorem 8. Let $x^{*}$ be a feasible point of (VQFP). Suppose that the constraint function $h_{j}$ is convex for each $j \in J$ and there exist vectors $\tau \in \mathbb{R}^{m}, \lambda \in \mathbb{R}^{\ell}$, such that (3), (4), and (5) are 
valid. Consider also, for each $i \in I$ and $k \in K \equiv\{1, \ldots, n\}$, the following functions:

$$
\begin{aligned}
\gamma_{i}^{k}\left(x, x^{*}, \tau\right) & \equiv \frac{\tau_{i}}{g_{i}\left(x^{*}\right)}\left\langle x-x^{*}, p_{i}^{k}\right\rangle^{2}, \\
\eta_{i}^{k}\left(x, x^{*}, \tau\right) & \equiv \frac{\tau_{i} f_{i}\left(x^{*}\right)}{\left[g_{i}\left(x^{*}\right)\right]^{2}}\left\langle x-x^{*}, q_{i}^{k}\right\rangle^{2},
\end{aligned}
$$

where $p_{i}^{k}$ and $q_{i}^{k}$ are the columns of orthogonal matrices $P_{i}$ and $Q_{i}$, constructed from the normalized eigenvectors of the matrices $A_{i}$ and $B_{i}$, respectively. If for all $x \in S$ the following inequality

$$
\mu_{k}^{A_{i}} \gamma_{i}^{k}\left(x, x^{*}, \tau\right) \geqq \mu_{k}^{B_{i}} \eta_{i}^{k}\left(x, x^{*}, \tau\right), \quad \forall i \in I, \quad \forall k \in K,
$$

is valid, where $\mu_{k}^{A_{i}}$ and $\mu_{k}^{B_{i}}$ are the eigenvalues of the matrices $A_{i}$ and $B_{i}$ associated with the eigenvectors $p_{i}^{k}$ and $q_{i}^{k}$, respectively, then $x^{*} \in \mathrm{Eff}(V Q F P)$.

Proof. The matrices $A_{i}$ and $B_{i}, i \in I$, are diagonalizable and can be rewritten as $A_{i}=P_{i} D_{A_{i}} P_{i}^{T}=\sum_{k=1}^{n} \mu_{k}^{A_{i}} p_{i}^{k} p_{i}^{k^{T}}$ and $B_{i}=$ $Q_{i} D_{B_{i}} Q_{i}^{T}=\sum_{k=1}^{n} \mu_{k}^{B_{i}} q_{i}^{k} q_{i}^{k^{T}}$, where $D_{A_{i}}$ and $D_{B_{i}}$ are diagonal matrices, with their diagonal formed by the eigenvalues $\mu_{k}^{A_{i}}$ and $\mu_{k}^{B_{i}}, k \in K$, of the matrices $A_{i}$ and $B_{i}$, respectively. Thus, we obtain

$$
\begin{aligned}
\sum_{i=1}^{m} \tau_{i} \frac{s_{i}\left(x, x^{*}\right)}{u_{i}\left(x, x^{*}\right)} & \\
=\sum_{i=1}^{m} \tau_{i} \frac{g_{i}(x)}{g_{i}\left(x^{*}\right)} \frac{1}{g_{i}(x)} & \cdot\left\{\left(x-x^{*}\right)^{T}\left[A_{i}-\frac{f_{i}\left(x^{*}\right)}{g_{i}\left(x^{*}\right)} B_{i}\right]\left(x-x^{*}\right)\right\} \\
= & \sum_{i=1}^{m}\left\{\left(x-x^{*}\right)^{T}\left[\frac{\tau_{i}}{g_{i}\left(x^{*}\right)} A_{i}-\frac{\tau_{i} f_{i}\left(x^{*}\right)}{\left[g_{i}\left(x^{*}\right)\right]^{2}} B_{i}\right]\left(x-x^{*}\right)\right\} \\
= & \sum_{i=1}^{m}\left\{( x - x ^ { * } ) ^ { T } \left[\frac{\tau_{i}}{g_{i}\left(x^{*}\right)}\left(\sum_{k=1}^{n} \mu_{k}^{A_{i}} p_{i}^{k} p_{i}^{k^{T}}\right)\right.\right. \\
= & \sum_{i=1}^{m}\left[\sum_{k=1}^{n} \mu_{k}^{A_{i}}\left(\frac{\tau_{i} f_{i}\left(x^{*}\right)}{\left.g_{i}\left(x^{*}\right)\right]^{2}}\left(\sum_{k=1}^{n} \mu_{k}^{B_{i}} q_{i}^{k} q_{i}^{k^{T}}\right)\right]\left(x-x^{*}\right)\right\} \\
= & -\sum_{i=1}^{n} \sum_{k=1}^{n} \mu_{k}^{B_{i}}\left(\frac{\tau_{i} f_{i}\left(x^{*}\right)}{\left[g_{i}\left(x^{*}\right)\right]^{2}}\left\langle\mu_{i}^{k}\right\rangle^{2}\right) \\
= & \left.\sum_{i=1}^{m}\left[\sum_{k=1}^{n} \mu_{k}^{A_{i}} \gamma_{i}^{k} \gamma_{i}^{k}\left(x, x^{*}, \tau\right)-\sum_{k=1}^{n} \mu_{k}^{B_{i}} \eta_{i}^{k}\left(x, x_{i}^{k}\right\rangle^{2}\right)\right]
\end{aligned}
$$

Since, for all $x \in S$, we have $\mu_{k}^{A_{i}} \gamma_{i}^{k}\left(x, x^{*}, \tau\right) \geqq \mu_{k}^{B_{i}} \eta_{i}^{k}\left(x, x^{*}, \tau\right)$, for all $i \in I$ and $k \in K$, we conclude that $Z\left(x, x^{*}\right) \geqq 0$. Therefore, inequality (6) is valid and the result follows from Theorem 5.

Theorem 8 is not simple to use since (26) depends on all points of the feasible set; that is, it depends on the functions $\gamma_{i}^{k}\left(x, x^{*}, \tau\right), \eta_{i}^{k}\left(x, x^{*}, \tau\right), \forall i \in I, \forall k \in K$, and $x \in S$. However, even if, for some $i \in I$ and $k \in K, \mu_{k}^{A_{i}} \gamma_{i}^{k}\left(x, x^{*}, \tau\right)<$ $\mu_{k}^{B_{i}} \eta_{i}^{k}\left(x, x^{*}, \tau\right)$ occurs, inequality (6) can still be satisfied. In order to obtain (26), we present the next corollary, which follows immediately from the previous theorem.

Corollary 9. Let $x^{*}$ be a feasible point of (VQFP). Suppose that the constraint function $h_{j}$ is convex for each $j \in J$ and there exist vectors $\tau \in \mathbb{R}^{m}, \lambda \in \mathbb{R}^{\ell}$, such that (3), (4), and (5) are valid. Consider also, for each $i \in I$ and $k \in K$,

$$
\begin{aligned}
a_{i, k}^{+} & \equiv \sqrt{\mu_{k}^{A_{i}}} p_{i}^{k}+\sqrt{\mu_{k}^{B_{i}} \frac{f_{i}\left(x^{*}\right)}{g_{i}\left(x^{*}\right)}} q_{i}^{k}, \\
a_{i, k}^{-} & \equiv \sqrt{\mu_{k}^{A_{i}}} p_{i}^{k}-\sqrt{\mu_{k}^{B_{i}} \frac{f_{i}\left(x^{*}\right)}{g_{i}\left(x^{*}\right)}} q_{i}^{k}, \\
\alpha_{i, k} & \equiv\left\langle x^{*}, a_{i, k}^{+}\right\rangle a_{i, k}^{-}+\left\langle x^{*}, a_{i, k}^{-}\right\rangle a_{i, k}^{+}, \\
\beta_{i, k} & \equiv\left\langle x^{*}, a_{i, k}^{+}\right\rangle\left\langle x^{*}, a_{i, k}^{-}\right\rangle, \\
H_{i, k}(x) & \equiv x^{T}\left[a_{i, k}^{+} a_{i, k}^{-T}\right] x-\alpha_{i, k}^{T} x+\beta_{i, k},
\end{aligned}
$$

where $p_{i}^{k}$ and $q_{i}^{k}$ are the columns of orthogonal matrices $P_{i}$ and $Q_{i}$, constructed from the normalized eigenvectors of the matrices $A_{i}, B_{i}$ and $\mu_{k}^{A_{i}}, \mu_{k}^{B_{i}}$ are the eigenvalues of the matrices $A_{i}$ and $B_{i}$ associated with the eigenvectors $p_{i}^{k}$ and $q_{i}^{k}$, respectively. If, for all $x \in S$, one obtains $H_{i, k}(x) \geqq 0$ for each $i \in I$ and $k \in K$, then $x^{*} \in \operatorname{Eff}(V Q F P)$.

Proof. According to Theorem 8, it is enough to show that, for every feasible point and for all $i \in I$ and $k \in K$, $\mu_{k}^{A_{i}} \gamma_{i}^{k}\left(x, x^{*}, \tau\right) \geqq \mu_{k}^{B_{i}} \eta_{i}^{k}\left(x, x^{*}, \tau\right)$ is valid. Given $x \in S$ and a pair $\{i, k\} \in I \times K$, we obtain

$$
\begin{aligned}
& \mu_{k}^{A_{i}} \gamma_{i}^{k}\left(x, x^{*}, \tau\right) \geqq \mu_{k}^{B_{i}} \eta_{i}^{k}\left(x, x^{*}, \tau\right) \\
& \Longleftrightarrow \frac{\tau_{i} \mu_{k}^{A_{i}}}{g_{i}\left(x^{*}\right)}\left\langle x-x^{*}, p_{i}^{k}\right\rangle^{2} \geqq \frac{\tau_{i} \mu_{k}^{B_{i}} f_{i}\left(x^{*}\right)}{\left[g_{i}\left(x^{*}\right)\right]^{2}}\left\langle x-x^{*}, q_{i}^{k}\right\rangle^{2} \\
& \Longleftrightarrow \mu_{k}^{A_{i}}\left\langle x-x^{*}, p_{i}^{k}\right\rangle^{2} \geqq \mu_{k}^{B_{i}} \frac{f_{i}\left(x^{*}\right)}{g_{i}\left(x^{*}\right)}\left\langle x-x^{*}, q_{i}^{k}\right\rangle^{2} \\
& \Longleftrightarrow\left(\sqrt{\mu_{k}^{A_{i}}}\left\langle x-x^{*}, p_{i}^{k}\right\rangle+\sqrt{\mu_{k}^{B_{i}} \frac{f_{i}\left(x^{*}\right)}{g_{i}\left(x^{*}\right)}}\left\langle x-x^{*}, q_{i}^{k}\right\rangle\right) \\
& \cdot\left(\sqrt{\mu_{k}^{A_{i}}}\left\langle x-x^{*}, p_{i}^{k}\right\rangle-\sqrt{\mu_{k}^{B_{i}} \frac{f_{i}\left(x^{*}\right)}{g_{i}\left(x^{*}\right)}}\left\langle x-x^{*}, q_{i}^{k}\right\rangle\right) \geqq 0
\end{aligned}
$$




$$
\begin{aligned}
& \Longleftrightarrow\left(\left\langle x-x^{*}, \sqrt{\mu_{k}^{A_{i}}} p_{i}^{k}+\sqrt{\mu_{k}^{B_{i}} \frac{f_{i}\left(x^{*}\right)}{g_{i}\left(x^{*}\right)}} q_{i}^{k}\right\rangle\right) \\
& \cdot\left(\left\langle x-x^{*}, \sqrt{\mu_{k}^{A_{i}}} p_{i}^{k}-\sqrt{\mu_{k}^{B_{i}} \frac{f_{i}\left(x^{*}\right)}{g_{i}\left(x^{*}\right)}} q_{i}^{k}\right\rangle\right) \geqq 0 \\
& \Longleftrightarrow\left(\left\langle x-x^{*}, a_{i, k}^{+}\right\rangle\right)\left(\left\langle x-x^{*}, a_{i, k}^{-}\right\rangle\right) \geqq 0 \\
& \Longleftrightarrow\left(\left\langle x, a_{i, k}^{+}\right\rangle-\left\langle x^{*}, a_{i, k}^{+}\right\rangle\right)\left(\left\langle x, a_{i, k}^{-}\right\rangle-\left\langle x^{*}, a_{i, k}^{-}\right\rangle\right) \geqq 0 \\
& \Longleftrightarrow\left\langle x, a_{i, k}^{+}\right\rangle\left\langle x, a_{i, k}^{-}\right\rangle-\left\langle x^{*}, a_{i, k}^{-}\right\rangle\left\langle x, a_{i, k}^{+}\right\rangle \\
& -\left\langle x^{*}, a_{i, k}^{+}\right\rangle\left\langle x, a_{i, k}^{-}\right\rangle+\left\langle x^{*}, a_{i, k}^{+}\right\rangle\left\langle x^{*}, a_{i, k}^{-}\right\rangle \geqq 0 \\
& \Longleftrightarrow\left\langle x, a_{i, k}^{+}\right\rangle\left\langle x, a_{i, k}^{-}\right\rangle \\
& -\left\langle x,\left(\left\langle x^{*}, a_{i, k}^{+}\right\rangle a_{i, k}^{-}+\left\langle x^{*}, a_{i, k}^{-}\right\rangle a_{i, k}^{+}\right)\right\rangle \\
& +\left\langle x^{*}, a_{i, k}^{+}\right\rangle\left\langle x^{*}, a_{i, k}^{-}\right\rangle \geqq 0 \\
& \Longleftrightarrow\left\langle x, a_{i, k}^{+}\right\rangle\left\langle x, a_{i, k}^{-}\right\rangle-\left\langle x, \alpha_{i, k}\right\rangle+\beta_{i, k} \geqq 0 \\
& \Longleftrightarrow x^{T}\left[a_{i, k}^{+} a_{i, k}^{-T}\right] x-\alpha_{i, k}^{T} x+\beta_{i, k} \geqq 0 \\
& \Longleftrightarrow H_{i, k}(x) \geqq 0 \text {. }
\end{aligned}
$$

Therefore, the result follows from Theorem 8 .

From Corollary 9, if each quadratic function $H_{i, k}(x)$, $\{i, k\} \in I \times K$, is nonnegative in the feasible set, then a feasible point satisfying (3), (4), and (5) is a Pareto optimal solution of (VQFP).

Let $\left[\bar{H}_{i, k}\right] \equiv\left[a_{i, k}^{+} a_{i, k}^{-T}\right] \in \mathbb{R}^{n \times n}$. Then $\beta_{i, k}=x^{* T}\left[\bar{H}_{i, k}\right] x^{*}$, and the nonnegativity of the quadratic $H_{i, k}(x)=x^{T}\left[\bar{H}_{i, k}\right] x-$ $\alpha_{i, k}^{T} x+\beta_{i, k}$ depends on each matrix $\left[\bar{H}_{i, k}\right]$ and each vector $\alpha_{i, k} \in \mathbb{R}^{n}$, where $\{i, k\} \in I \times K$ and $x^{*} \in S$. For example, the unconstrained (VQFP) requires that each matrix $\left[\bar{H}_{i, k}\right]$ be positive semidefinite and that $\beta_{i, k} \geqq\left(\alpha_{i, k}-w^{T}\left[\bar{H}_{i, k}\right] w\right)$, where $w$ is a solution of the system $2\left[\bar{H}_{i, k}\right] x=\alpha_{i, k}$.

Corollary 10. Let $x^{*}$ be a feasible point of (VQFP). Suppose that the constraint function $h_{j}$ is convex for each $j \in J$ and there exist vectors $\tau \in \mathbb{R}^{m}, \lambda \in \mathbb{R}^{\ell}$, such that (3), (4), and (5) are valid. If, for each pair $\{i, k\} \in I \times K$, the matrix $\left[\bar{H}_{i, k}\right]$ is positive semidefinite and $\alpha_{i, k}=0$ (see (28)), then $x^{*} \in \operatorname{Eff}(V Q F P)$.

Proof. By hypothesis, for all $x \in S$, we have $H_{i, k}(x) \geqq 0$ for each pair $\{i, k\} \in I \times K$. Therefore, the result follows from Corollary 9.

Given a pair $\{i, k\} \in I \times K$, writing each entry of the matrix $\left[\bar{H}_{i, k}\right]=\left(\bar{H}_{i, k}(r, s)\right)$ and each entry of the vector $\alpha_{i, k}=\left(\alpha_{i, k}(r)\right)$ according to the entries of the eigenvectors $p_{i}^{k}=\left(p_{i}^{k}(r)\right)$ and $q_{i}^{k}=\left(q_{i}^{k}(r)\right)$, where $r, s \in K$, we obtain, for each pair $\{r, s\} \in K \times K$,

$$
\begin{aligned}
& \bar{H}_{i, k}(r, s)= a_{i, k}^{+}(r) a_{i, k}^{-}(s) \\
&= \mu_{k}^{A_{i}} p_{i}^{k}(r) p_{i}^{k}(s) \\
&+\sqrt{\mu_{k}^{A_{i}} \mu_{k}^{B_{i}} \frac{f_{i}\left(x^{*}\right)}{g_{i}\left(x^{*}\right)}}\left(p_{i}^{k}(s) q_{i}^{k}(r)-p_{i}^{k}(r) q_{i}^{k}(s)\right) \\
&-\mu_{k}^{B_{i}} \frac{f_{i}\left(x^{*}\right)}{g_{i}\left(x^{*}\right)} q_{i}^{k}(r) q_{i}^{k}(s), \\
& \bar{H}_{i, k}(r, r)=\mu_{k}^{A_{i}}\left(p_{i}^{k}(r)\right)^{2}-\mu_{k}^{B_{i}} \frac{f_{i}\left(x^{*}\right)}{g_{i}\left(x^{*}\right)}\left(q_{i}^{k}(r)\right)^{2}, \\
& \alpha_{i, k}(r)=2 \mu_{k}^{A_{i}}\left\langle x^{*}, p_{i}^{k}\right\rangle p_{i}^{k}(r)-2 \mu_{k}^{B_{i}} \frac{f_{i}\left(x^{*}\right)}{g_{i}\left(x^{*}\right)}\left\langle x^{*}, q_{i}^{k}\right\rangle q_{i}^{k}(r) .
\end{aligned}
$$

We can draw some conclusions from (30). For example, for a fixed pair $\{i, k\} \in I \times K$, the vector $\alpha_{i, k}$ is a linear combination of the eigenvectors $p_{i}^{k}$ and $q_{i}^{k}$. If $\mu_{k}^{A_{i}}=0, \mu_{k}^{B_{i}}=0$, or $f_{i}\left(x^{*}\right)=0$, then $\left[\bar{H}_{i, k}\right]$ is a symmetric matrix. Moreover, if $\mu_{k}^{A_{i}} \mu_{k}^{B_{i}} f_{i}\left(x^{*}\right)<0$ and there exists a pair $\{r, s\} \in K \times K$ such that $p_{i}^{k}(s) q_{i}^{k}(r) \neq p_{i}^{k}(r) q_{i}^{k}(s)$, then the matrix $\left[\bar{H}_{i, k}\right] \notin \mathbb{R}^{n \times n}$. In this case, if there exists $x \in S$ such that $H_{i, k}(x) \in \mathbb{C} \backslash \mathbb{R}$, $H_{i, k}(x) \geqq 0$ does not make sense. However, when (26) is required, it is possible to show that $H_{i, k}(x) \in \mathbb{C} \backslash \mathbb{R}$ is not possible.

The results of Theorems 5 and 8 and their corollaries can be used in order to develop a method of searching for Pareto optimal solutions of (VQFP), and it might be useful to determine the termination criteria in the development of algorithms.

\section{Duality}

Matrix (24) defines a specific function, and by adding some assumptions about it, we obtain new results, such as, a relationship between the problem (VQFP) and a scalar problem associated with it, and the main duality theorems.

In the scalar optimization problem case, Dinkelbach [3] and Jagannathan [4] used a parametric approach that transforms the fractional optimization problem in a new scalar optimization problem. Similarly, we consider the following parameterized problem $(\mathrm{VQFP})_{x^{*}}$ associated with the problem (VQFP):

$$
\begin{aligned}
\text { Minimize } f(x) & -\frac{f\left(x^{*}\right)}{g\left(x^{*}\right)} g(x) \\
= & \left(f_{1}(x)-\frac{f_{1}\left(x^{*}\right)}{g_{1}\left(x^{*}\right)} g_{1}(x), \ldots, f_{m}(x)\right. \\
& \left.\quad-\frac{f_{m}\left(x^{*}\right)}{g_{m}\left(x^{*}\right)} g_{m}(x)\right)
\end{aligned}
$$

subject to $h_{j}(x) \leqq 0 \quad j \in J, x \in \Omega$, 
where $\Omega \subseteq \mathbb{R}^{n}, f_{i}, g_{i}, i \in I$, and $h_{j}, j \in J$, are defined in (VQFP), and $x^{*} \in S$.

Using assumptions of generalized convexity, OsunaGómez et al. [17] presented the problem $(\mathrm{VFP})_{x^{*}}$ and obtained necessary and sufficient conditions for weakly Pareto optimality and main duality theorems. The results presented in $[3,4,17]$ considered each objective function as $f_{i}(x)-\alpha_{i} g_{i}(x), i \in I$, and they studied the properties of the parameter $\alpha_{i} \in \mathbb{R}$. Following the ideas presented by OsunaGómez et al. [17], we obtain new results by considering directly $\alpha_{i} \equiv f_{i}\left(x^{*}\right) / g_{i}\left(x^{*}\right), i \in I$, where $x^{*} \in$ Eff (VQFP). However, by imposing hypothesis on the linear combination of matrices $\left[A_{i}-\left(f_{i}\left(x^{*}\right) / g_{i}\left(x^{*}\right)\right) B_{i}\right], i \in I$ and $x^{*} \in S$, we consider Pareto optimal solutions rather than weakly Pareto optimal solutions.

To characterize the solutions of the problems (VOP), Geoffrion [37] used the solutions of the associated scalar problems. Similarly, we consider the following weighted scalar problem $(\mathrm{VQFP})_{x^{*}}^{w}$ associated with the problem (VQFP):

$$
\begin{array}{ll}
\text { Minimize } & \sum_{i=1}^{m} w_{i}\left(f_{i}(x)-\frac{f_{i}\left(x^{*}\right)}{g_{i}\left(x^{*}\right)} g_{i}(x)\right) \quad(\mathrm{VQFP})_{x^{*}}^{w} \\
\text { subject to } & h_{j}(x) \leqq 0 \quad j \in J, x \in \Omega,
\end{array}
$$

where $\Omega \subseteq \mathbb{R}^{n}, f_{i}, g_{i}, i \in I$, and $h_{j}, j \in J$, are defined in (VQFP), $x^{*} \in S$ and $w=\left(w_{1}, \ldots, w_{m}\right)^{T} \in \mathbb{R}^{m}, w>0$.

4.1. The Relationship between the Associated Problems. The next theorem and its proof are similar to Lemma 1.1 from [17], when Pareto optimal solutions (not necessarily weak) are considered.

Theorem 11. $x^{*} \in \mathrm{Eff}(V Q F P)$ if and only if $x^{*} \in$ $\mathrm{Eff}(V Q F P)_{x^{*}}$.

Proof. See Lemma 1.1 in [17], considering " $\leq$ " instead of "<."

In Section 3, we define the matrix $\widehat{F}\left(x^{*}\right)=$ $\sum_{i=1}^{m}\left[\left(\tau_{i} / g_{i}\left(x^{*}\right)\right) A_{i}-\left(\tau_{i} f_{i}\left(x^{*}\right) /\left[g_{i}\left(x^{*}\right)\right]^{2}\right) B_{i}\right]$, where $x^{*} \in S$ and $\tau \in \mathbb{R}^{m}, \tau>0$. Let us define now the set $\mathscr{W}=\left\{w \in \mathbb{R}^{m} \mid w>0\right\}$, the function $F: \mathscr{W} \times S \rightarrow \mathbb{R}^{n \times n}$ given by

$$
F(w, x) \equiv \sum_{i=1}^{m} w_{i}\left[A_{i}-\frac{f_{i}(x)}{g_{i}(x)} B_{i}\right],
$$

and, for each $i \in I$, the functions $F_{i}: S \rightarrow \mathbb{R}^{n \times n}$ given by

$$
F_{i}(x) \equiv A_{i}-\frac{f_{i}(x)}{g_{i}(x)} B_{i} .
$$

Then, we have $\widehat{F}\left(x^{*}\right)=F\left(\tau / g\left(x^{*}\right), x^{*}\right)$, where $\tau / g\left(x^{*}\right)=$ $\left(\tau_{1} / g_{1}\left(x^{*}\right), \ldots, \tau_{m} / g_{m}\left(x^{*}\right)\right)^{T} \in \mathscr{W}, F(w, x)=\sum_{i=1}^{m} w_{i} F_{i}(x)$, and we can establish some relations among the associated problems (VQFP), (VQFP) $)_{x^{*}}$, and (VQFP) $)_{x^{*}}^{w}$.

Theorem 12. If $x^{*}$ is an optimal solution of the weighted scalar problem $(V Q F P)_{x^{*}}^{w}$, then $x^{*} \in \mathrm{Eff}(V Q F P)$.
Proof. Suppose that $x^{*} \notin$ Eff (VQFP); then there exists another point $x \in S$ such that

$$
\begin{aligned}
\frac{f_{i}(x)}{g_{i}(x)} & \leq \frac{f_{i}\left(x^{*}\right)}{g_{i}\left(x^{*}\right)} \\
& \Longrightarrow f_{i}(x)-\frac{f_{i}\left(x^{*}\right)}{g_{i}\left(x^{*}\right)} g_{i}(x) \leq 0 \\
& \Longrightarrow \sum_{i=1}^{m} w_{i}\left(f_{i}(x)-\frac{f_{i}\left(x^{*}\right)}{g_{i}\left(x^{*}\right)} g_{i}(x)\right)<0 \\
& \Longrightarrow \sum_{i=1}^{m} w_{i}\left(f_{i}(x)-\frac{f_{i}\left(x^{*}\right)}{g_{i}\left(x^{*}\right)} g_{i}(x)\right) \\
& <\sum_{i=1}^{m} w_{i}\left(f_{i}\left(x^{*}\right)-\frac{f_{i}\left(x^{*}\right)}{g_{i}\left(x^{*}\right)} g_{i}\left(x^{*}\right)\right) .
\end{aligned}
$$

This contradicts the minimality of $x^{*}$ in $(\mathrm{VQFP})_{x^{*}}^{w}$.

Lemma 13. Let $x^{*} \in \mathrm{Eff}(V Q F P)$. Suppose that the constraint qualification (GGCQ) is satisfied at $x^{*}$; then there exist vectors $\tau^{*}>0$ and $\lambda^{*} \geqq 0$ such that

$$
\begin{gathered}
\sum_{i=1}^{m} \tau_{i}^{*}\left(\nabla f_{i}\left(x^{*}\right)-\frac{f_{i}\left(x^{*}\right)}{g_{i}\left(x^{*}\right)} \nabla g_{i}\left(x^{*}\right)\right)+\sum_{j=1}^{\ell} \lambda_{j}^{*} \nabla h_{j}\left(x^{*}\right)=0, \\
\sum_{j=1}^{\ell} \lambda_{j}^{*} h_{j}\left(x^{*}\right)=0 .
\end{gathered}
$$

Proof. Let $x^{*} \in S, \mu \in \mathbb{R}^{m}, \mu>0$, and $\tau_{i}=\mu_{i} / g_{i}\left(x^{*}\right)>0$, $i \in I$. Then

$$
\begin{aligned}
& \sum_{i=1}^{m} \mu_{i} \nabla \frac{f_{i}\left(x^{*}\right)}{g_{i}\left(x^{*}\right)}=\sum_{i=1}^{m} \tau_{i} g_{i}\left(x^{*}\right) \nabla \frac{f_{i}\left(x^{*}\right)}{g_{i}\left(x^{*}\right)} \\
& =\sum_{i=1}^{m} \tau_{i} g_{i}\left(x^{*}\right)\left(\frac{\nabla f_{i}\left(x^{*}\right) g_{i}\left(x^{*}\right)-\nabla g_{i}\left(x^{*}\right) f_{i}\left(x^{*}\right)}{\left[g_{i}\left(x^{*}\right)\right]^{2}}\right) \\
& =\sum_{i=1}^{m} \tau_{i}\left(\nabla f_{i}\left(x^{*}\right)-\frac{f_{i}\left(x^{*}\right)}{g_{i}\left(x^{*}\right)} \nabla g_{i}\left(x^{*}\right)\right),
\end{aligned}
$$

and if $x^{*} \in$ Eff (VQFP), by Lemma 4 , there exist $\mu^{*}>0$ and $\lambda^{*} \geqq 0$ such that $\left(x^{*}, \mu^{*}, \lambda^{*}\right)$ is a critical point, in the KKT sense, of the problem (VQFP). That is,

$$
\begin{aligned}
\sum_{i=1}^{m} \mu_{i}^{*} \nabla \frac{f_{i}\left(x^{*}\right)}{g_{i}\left(x^{*}\right)}+\sum_{j=1}^{\ell} \lambda_{j}^{*} \nabla h_{j}\left(x^{*}\right) & =0, \\
\sum_{j=1}^{\ell} \lambda_{j}^{*} h_{j}\left(x^{*}\right) & =0 .
\end{aligned}
$$


From (35), there exist $\tau^{*}>0, \tau_{i}^{*}=\mu_{i}^{*} / g_{i}\left(x^{*}\right)>0, i \in I$, and $\lambda^{*} \geqq 0$ such that

$$
\begin{gathered}
\sum_{i=1}^{m} \tau_{i}^{*}\left(\nabla f_{i}\left(x^{*}\right)-\frac{f_{i}\left(x^{*}\right)}{g_{i}\left(x^{*}\right)} \nabla g_{i}\left(x^{*}\right)\right)+\sum_{j=1}^{\ell} \lambda_{j}^{*} \nabla h_{j}\left(x^{*}\right)=0, \\
\sum_{j=1}^{\ell} \lambda_{j}^{*} h_{j}\left(x^{*}\right)=0 .
\end{gathered}
$$

Therefore, the result is valid.

Lemma 14. Let $x^{*} \in S$. If there exists $w \in \mathscr{W}$, such that the matrix $F\left(w, x^{*}\right)$ is positive semidefinite, then the objective function of $(V Q F P)_{x^{*}}^{w}$ is convex.

Proof. Given $x_{1}, x_{2} \in S$, we have, for each $i \in I$,

$$
\begin{aligned}
f_{i}\left(x_{1}\right)-f_{i}\left(x_{2}\right)= & \left(x_{1}-x_{2}\right)^{T} A_{i}\left(x_{1}-x_{2}\right) \\
& +\nabla f_{i}\left(x_{2}\right)^{T}\left(x_{1}-x_{2}\right), \\
g_{i}\left(x_{1}\right)-g_{i}\left(x_{2}\right)= & \left(x_{1}-x_{2}\right)^{T} B_{i}\left(x_{1}-x_{2}\right) \\
& +\nabla g_{i}\left(x_{2}\right)^{T}\left(x_{1}-x_{2}\right) .
\end{aligned}
$$

Hence, for each objective function of $(\mathrm{VQFP})_{x^{*}}$, we have

$$
\begin{aligned}
\left(f_{i}\left(x_{1}\right)-\frac{f_{i}\left(x^{*}\right)}{g_{i}\left(x^{*}\right)} g_{i}\left(x_{1}\right)\right) & -\left(f_{i}\left(x_{2}\right)-\frac{f_{i}\left(x^{*}\right)}{g_{i}\left(x^{*}\right)} g_{i}\left(x_{2}\right)\right) \\
= & \left(f_{i}\left(x_{1}\right)-f_{i}\left(x_{2}\right)\right)-\frac{f_{i}\left(x^{*}\right)}{g_{i}\left(x^{*}\right)}\left(g_{i}\left(x_{1}\right)-g_{i}\left(x_{2}\right)\right) \\
= & \left\{\left(x_{1}-x_{2}\right)^{T} A_{i}\left(x_{1}-x_{2}\right)+\nabla f_{i}\left(x_{2}\right)^{T}\left(x_{1}-x_{2}\right)\right\} \\
& -\frac{f_{i}\left(x^{*}\right)}{g_{i}\left(x^{*}\right)} \\
& \cdot\left\{\left(x_{1}-x_{2}\right)^{T} B_{i}\left(x_{1}-x_{2}\right)+\nabla g_{i}\left(x_{2}\right)^{T}\left(x_{1}-x_{2}\right)\right\} \\
= & \left(x_{1}-x_{2}\right)^{T}\left[A_{i}-\frac{f_{i}\left(x^{*}\right)}{g_{i}\left(x^{*}\right)} B_{i}\right]\left(x_{1}-x_{2}\right) \\
& +\left(\nabla f_{i}\left(x_{2}\right)-\frac{f_{i}\left(x^{*}\right)}{g_{i}\left(x^{*}\right)} \nabla g_{i}\left(x_{2}\right)\right)^{T}\left(x_{1}-x_{2}\right) .
\end{aligned}
$$

If there exists $w \in \mathscr{W}$ such that the matrix $F\left(w, x^{*}\right)$ is positive semidefinite, then

$$
\begin{aligned}
& \sum_{i=1}^{m} w_{i}\left(f_{i}\left(x_{1}\right)-\frac{f_{i}\left(x^{*}\right)}{g_{i}\left(x^{*}\right)} g_{i}\left(x_{1}\right)\right) \\
& -\sum_{i=1}^{m} w_{i}\left(f_{i}\left(x_{2}\right)-\frac{f_{i}\left(x^{*}\right)}{g_{i}\left(x^{*}\right)} g_{i}\left(x_{2}\right)\right)
\end{aligned}
$$

$$
\begin{aligned}
= & \left(x_{1}-x_{2}\right)^{T} \sum_{i=1}^{m} w_{i}\left[A_{i}-\frac{f_{i}\left(x^{*}\right)}{g_{i}\left(x^{*}\right)} B_{i}\right]\left(x_{1}-x_{2}\right) \\
& +\sum_{i=1}^{m} w_{i}\left(\nabla f_{i}\left(x_{2}\right)-\frac{f_{i}\left(x^{*}\right)}{g_{i}\left(x^{*}\right)} \nabla g_{i}\left(x_{2}\right)\right)^{T}\left(x_{1}-x_{2}\right) \\
\geqq & \left(\sum_{i=1}^{m} w_{i}\left(\nabla f_{i}\left(x_{2}\right)-\frac{f_{i}\left(x^{*}\right)}{g_{i}\left(x^{*}\right)} \nabla g_{i}\left(x_{2}\right)\right)\right)^{T}\left(x_{1}-x_{2}\right) .
\end{aligned}
$$

Therefore, the objective function of $(\mathrm{VQFP})_{x^{*}}^{w}$ is convex.

Note that the hypothesis of semidefiniteness on the matrix $F\left(w, x^{*}\right)$ or on the matrices $F_{i}\left(x^{*}\right), i \in I, x^{*} \in S$, is punctual. However, in the next example, we draw a situation in which, for all $x \in S$ and $i \in I$, we have $y^{T} F_{i}(x) y \geqq 0$, for all $y \in S$, and then $y^{T} F(w, x) y \geqq 0$, for all $y \in S$.

Example. Consider the problem (VQFP), where $S=[-2,2]$ and for all $x \in S$

$$
\begin{aligned}
& \frac{f_{1}(x)}{g_{1}(x)}=\frac{x-2}{x^{2}+2} \\
& \frac{f_{2}(x)}{g_{2}(x)}=\frac{2 x^{2}-x-1}{x^{2}+1} \\
& \frac{f_{3}(x)}{g_{3}(x)}=\frac{-2 x^{2}-2 x-5}{x^{2}+x+1}
\end{aligned}
$$

For all these functions, we obtain for all $y \in S$

$$
\begin{aligned}
y^{T}\left[A_{1}-\frac{f_{1}(x)}{g_{1}(x)} B_{1}\right] y & =y^{2}\left(0-\frac{x-2}{x^{2}+2} 1\right) \\
& =\frac{y^{2}(2-x)}{x^{2}+2} \geqq 0, \\
y^{T}\left[A_{2}-\frac{f_{2}(x)}{g_{2}(x)} B_{2}\right] y & =y^{2}\left(2-\frac{2 x^{2}-x-1}{x^{2}+1} 1\right) \\
& =\frac{y^{2}(x+3)}{x^{2}+1} \geqq 0, \\
y^{T}\left[A_{3}-\frac{f_{3}(x)}{g_{3}(x)} B_{3}\right] y & =y^{2}\left(-2-\frac{-2 x^{2}-2 x-5}{x^{2}+x+1} 1\right) \\
& =\frac{3 y^{2}}{x^{2}+x+1} \geqq 0 .
\end{aligned}
$$

Therefore, for this example, each point $x^{*}$ satisfying (3), (4), and (5) is Pareto optimal. For example, for $\left(\tau_{1}, \tau_{2}, \tau_{3}\right)^{T}=$ $(0.5,1,0.25)^{T}$ and $\left(\lambda_{1}, \lambda_{2}\right)^{T}=(1,1)$, we have that $x^{*}=$ 0 is Pareto optimal solution. Likewise, for $\left(\tau_{1}, \tau_{2}, \tau_{3}\right)^{T} \approx$ $(0.62,1,0.89)^{T}$ and $\left(\lambda_{1}, \lambda_{2}\right)^{T}=(0,0)^{T}$, we have that $x^{*}=$ -0.25 is Pareto optimal solution.

Theorem 11 shows an equivalence between the associated problems (VQFP) and (VQFP) $)_{x^{*}}$. The next theorem shows a relation between the problems $(\mathrm{VQFP})_{x^{*}}$ and (VQFP) $)_{x^{*}}^{w}$; then it provides a converse to the Theorem 12 . 
Theorem 15. Let $x^{*} \in \operatorname{Eff}(V Q F P)_{x^{*}}$. Suppose that the constraint qualification (GGCQ) is satisfied at $x^{*}$ and the constraint function $h_{j}$ is convex for each $j \in J$. Then there exists $w \in \mathscr{W}$ such that if the matrix $F\left(w, x^{*}\right)$ is positive semidefinite, then $x^{*}$ is the optimal solution for the weighted scalar problem $(V Q F P)_{x^{*}}^{w}$.

Proof. If $x^{*} \in$ Eff(VQFP) $x^{*}$ and satisfies (GGCQ), by Lemma 13 , there exist $w>0$ and $\lambda \geqq 0$, such that

$$
\begin{gathered}
\sum_{i=1}^{m} w_{i}^{*}\left(\nabla f_{i}\left(x^{*}\right)-\frac{f_{i}\left(x^{*}\right)}{g_{i}\left(x^{*}\right)} \nabla g_{i}\left(x^{*}\right)\right)+\sum_{j=1}^{\ell} \lambda_{j}^{*} \nabla h_{j}\left(x^{*}\right)=0, \\
\sum_{j=1}^{\ell} \lambda_{j}^{*} h_{j}\left(x^{*}\right)=0 .
\end{gathered}
$$

Therefore, $x^{*}$ is a critical point of the weighted scalar problem $(\mathrm{VQFP})_{x^{*}}^{w}$, and since $F\left(w, x^{*}\right)$ is positive semidefinite, by Lemma 14, the objective function of (VQFP) $)_{x^{*}}^{w}$ is convex. Since for each $j \in J$ the constraint function $h_{j}$ is convex, it follows that $x^{*}$ is an optimal solution for (VQFP) $)_{x^{*}}^{w}$.

4.2. Duality Theorems. For a given mathematical optimization problem there are many types of duality. Two wellknown duals are the Wolfe dual [38] and the Mond-Weir dual [13]. In this work, we consider the primal problem (VQFP) and discuss the Mond-Weir dual problem, but we use the associated problem (VQFP) $x^{*}$ to generate the constraint set of the dual problem. Let us consider the following vector quadratic fractional dual optimization problem (VQFD):

$$
\begin{gathered}
\text { Maximize } \frac{f(u)}{g(u)}=\left(\frac{f_{1}(u)}{g_{1}(u)}, \ldots, \frac{f_{m}(u)}{g_{m}(u)}\right) \\
\text { subject to } \sum_{i=1}^{m} \tau_{i}\left(\nabla f_{i}(u)-\frac{f(u)}{g(u)} \nabla g_{i}(u)\right) \\
+\sum_{j=1}^{\ell} \lambda_{j} \nabla h_{j}(u)=0, \\
\sum_{j=1}^{\ell} \lambda_{j} h_{j}(u) \geqq 0, \\
\tau>0, \quad \lambda \geqq 0, \quad \sum_{j=1}^{\ell} \lambda_{j}=1,
\end{gathered}
$$$$
u \in S
$$

(VQFD)

where $f_{i}$ and $g_{i}, i \in I$, are the same quadratic functions defined on (VQFP), and we denote its feasible set by $Y$.

Theorem 16 (weak duality). Let $x \in S$ and $(u, \tau, \lambda) \in Y$. If $F(\tau, u)$ is positive semidefinite and the constraint function $h_{j}$ is convex for each $j \in J$, then

$$
\frac{f(x)}{g(x)} \nless \frac{f(u)}{g(u)} .
$$

Proof. If there are $x \in S$ and $(u, \tau, \lambda) \in Y$ such that $f(x) / g(x) \leq f(u) / g(u)$, then

$$
\begin{aligned}
\frac{f(x)}{g(x)} & \leq \frac{f(u)}{g(u)} \Longrightarrow f(x)-\frac{f(u)}{g(u)} g(x) \leq 0 \\
& \Longrightarrow \sum_{i=1}^{m} \tau_{i}\left(f_{i}(x)-\frac{f_{i}(u)}{g_{i}(u)} g_{i}(x)\right)<0 .
\end{aligned}
$$

Since $x \in S$, then $\sum_{j=1}^{\ell} \lambda_{j} h_{j}(x) \leqq 0$, and $\sum_{j=1}^{\ell} \lambda_{j} h_{j}(u) \geqq 0$ implies that

$$
\begin{aligned}
& \sum_{i=1}^{m} \tau_{i}\left(f_{i}(x)-\frac{f_{i}(u)}{g_{i}(u)} g_{i}(x)\right)+\sum_{j=1}^{\ell} \lambda_{j} h_{j}(x) \\
& \quad<\sum_{i=1}^{m} \tau_{i}\left(f_{i}(u)-\frac{f_{i}(u)}{g_{i}(u)} g_{i}(u)\right)+\sum_{j=1}^{\ell} \lambda_{j} h_{j}(u) .
\end{aligned}
$$

Once $F(\tau, u)$ is positive semidefinite and each constraint function $h_{j}$ is convex, we can use Lemma 14 to conclude that the objective function of $(\mathrm{VQFP})_{x^{*}}^{\tau}$ is convex, and

$$
\begin{aligned}
0> & \sum_{i=1}^{m} \tau_{i}\left[\left(f_{i}(x)-\frac{f_{i}(u)}{g_{i}(u)} g_{i}(x)\right)-\left(f_{i}(u)-\frac{f_{i}(u)}{g_{i}(u)} g_{i}(u)\right)\right] \\
& +\sum_{j=1}^{\ell} \lambda_{j}\left(h_{j}(x)-h_{j}(u)\right) \\
\geqq & \sum_{i=1}^{m} \tau_{i}\left(\nabla f_{i}(u)-\frac{f_{i}(u)}{g_{i}(u)} \nabla g_{i}(u)\right)^{T}(x-u) \\
& +\sum_{j=1}^{\ell} \lambda_{j} \nabla h_{j}(u)^{T}(x-u) \\
= & (x-u)^{T} \\
& \cdot\left(\sum_{i=1}^{m} \tau_{i}\left(\nabla f_{i}(u)-\frac{f_{i}(u)}{g_{i}(u)} \nabla g_{i}(u)\right)+\sum_{j=1}^{\ell} \lambda_{j} \nabla h_{j}(u)\right) \\
= & 0,
\end{aligned}
$$

which is a contradiction.

Theorem 17 (strong duality). Let $x^{*} \in \mathrm{Eff}(V Q F P)$. Suppose that (GGCQ) holds at $x^{*}$; then there exists $\left(\tau^{*}, \lambda^{*}\right)$ such that $\left(x^{*}, \tau^{*}, \lambda^{*}\right)$ is feasible for (VQFD) and the values of the objective function of (VQFP) and (VQFD) are equal. Moreover, if $F\left(\tau^{*}, x^{*}\right)$ is positive semidefinite and the constraint function $h_{j}$ is convex for each $j \in J$, then $\left(x^{*}, \tau^{*}, \lambda^{*}\right) \in \operatorname{Eff}(V Q F D)$. 
Proof. If $x^{*} \in$ Eff(VQFP), by Lemma 13, there are $\tau^{*}>0$ and $\lambda^{*} \geqq 0$ such that $\left(x^{*}, \tau^{*}, \lambda^{*}\right)$ satisfies

$$
\begin{gathered}
\sum_{i=1}^{m} \tau_{i}^{*}\left(\nabla f_{i}\left(x^{*}\right)-\frac{f_{i}\left(x^{*}\right)}{g_{i}\left(x^{*}\right)} \nabla g_{i}\left(x^{*}\right)\right)+\sum_{j=1}^{\ell} \lambda_{j}^{*} \nabla h_{j}\left(x^{*}\right)=0, \\
\sum_{j=1}^{\ell} \lambda_{j}^{*} h_{j}\left(x^{*}\right)=0 .
\end{gathered}
$$

Then $\left(x^{*}, \tau^{*}, \lambda^{*}\right) \in Y$ and the values of the objective functions of (VQFP) and (VQFD) are equal. Moreover, if $F\left(\tau^{*}, x^{*}\right)$ is positive semidefinite, each constraint function $h_{j}$ is convex, and $\left(x^{*}, \tau^{*}, \lambda^{*}\right) \notin \mathrm{Eff}(\mathrm{VQFD})$, then there exists another point $(u, \tau, \lambda) \in Y$ such that

$$
\frac{f(u)}{g(u)} \geq \frac{f\left(x^{*}\right)}{g\left(x^{*}\right)},
$$

contradicting the weak duality.

Theorem 18 (converse duality). Let $\left(u^{*}, \tau^{*}, \lambda^{*}\right) \in Y$ and $u^{*}$ be feasible point of the primal problem (VQFP). If $F\left(\tau^{*}, u^{*}\right)$ is positive semidefinite and the constraint function $h_{j}$ is convex for each $j \in J$, then $u^{*} \in \operatorname{Eff}(V Q F P)$.

Proof. If $\left(u^{*}, \tau^{*}, \lambda^{*}\right) \in Y$ and $u^{*} \in S$, then $\sum_{j=1}^{\ell} \lambda_{j}^{*} h_{j}\left(u^{*}\right) \geqq 0$, $\sum_{j=1}^{\ell} \lambda_{j}^{*} h_{j}\left(u^{*}\right) \leqq 0$, and

$$
\begin{gathered}
\sum_{i=1}^{m} \tau_{i}^{*}\left(\nabla f_{i}\left(u^{*}\right)-\frac{f_{i}\left(u^{*}\right)}{g_{i}\left(u^{*}\right)} \nabla g_{i}\left(u^{*}\right)\right)+\sum_{j=1}^{\ell} \lambda_{j}^{*} \nabla h_{j}\left(u^{*}\right)=0 \\
\sum_{j=1}^{\ell} \lambda_{j}^{*} h_{j}\left(u^{*}\right)=0
\end{gathered}
$$

Therefore, $u^{*}$ is a critical point for the weighted scalar problem (VQFP) ${ }_{u^{*}}^{\tau^{*}}$. Since $F\left(\tau^{*}, u^{*}\right)$ is positive semidefinite, by Lemma 14, the objective function of (VQFP) $)_{u^{*}}^{\tau^{*}}$ is convex. Moreover, if each constraint function $h_{j}$ is convex, $j \in J$, then $u^{*}$ is an optimal solution of (VQFP) $u^{\tau^{*}}$. Thus, by Theorem 12, we have $u^{*} \in$ Eff (VQFP).

We can obtain a second type of converse duality theorem requiring more of the matrix function $F$. Specifically, there must be vectors $(w, x) \in \mathscr{W} \times S$ such that $F(w, x)$ is positive definite; that is, $y^{T} F(w, x) y>0, \forall y \in \mathbb{R}^{n}$, and $y \neq 0$.

Theorem 19 (strict converse duality). Let $x^{*} \in S$ and $\left(u^{*}, \tau^{*}, \lambda^{*}\right) \in Y$ such that

$$
\sum_{i=1}^{m} \tau_{i}^{*} \frac{f_{i}\left(x^{*}\right)}{g_{i}\left(x^{*}\right)}=\sum_{i=1}^{m} \tau_{i}^{*} \frac{f_{i}\left(u^{*}\right)}{g_{i}\left(u^{*}\right)} .
$$

If the matrix $F\left(\tau^{*} / g\left(x^{*}\right), u^{*}\right)$ is positive definite and the constraint function $h_{j}$ is convex for each $j \in J$, then $x^{*}=u^{*}$.
Proof. Suppose $x^{*} \neq u^{*}$. Since $x^{*} \in S$ and $\left(u^{*}, \tau^{*}, \lambda^{*}\right) \in Y$, then $-\sum_{j=1}^{\ell} \lambda_{j}^{*} h_{j}\left(u^{*}\right) \leqq 0$ and $\sum_{j=1}^{\ell} \lambda_{j}^{*} h_{j}\left(x^{*}\right) \leqq 0$. If each constraint function $h_{j}$ is convex, $j \in J$, we obtain

$$
\begin{aligned}
0 & \geqq \sum_{j=1}^{\ell} \lambda_{j}^{*} h_{j}\left(x^{*}\right)-\sum_{j=1}^{\ell} \lambda_{j}^{*} h_{j}\left(u^{*}\right) \\
& \geqq\left(\sum_{j=1}^{\ell} \lambda_{j}^{*} \nabla h_{j}\left(u^{*}\right)\right)^{T}\left(x^{*}-u^{*}\right) \\
& =\left(-\sum_{i=1}^{m} \tau_{i}^{*}\left(\nabla f_{i}\left(u^{*}\right)-\frac{f_{i}\left(u^{*}\right)}{g_{i}\left(u^{*}\right)} \nabla g_{i}\left(u^{*}\right)\right)\right)^{T}\left(x^{*}-u^{*}\right) \\
& \Longrightarrow \sum_{i=1}^{m} \tau_{i}^{*}\left(\nabla f_{i}\left(u^{*}\right)-\frac{f_{i}\left(u^{*}\right)}{g_{i}\left(u^{*}\right)} \nabla g_{i}\left(u^{*}\right)\right)^{T}\left(x^{*}-u^{*}\right) \geqq 0 .
\end{aligned}
$$

Using the proof of Theorem 5 , given $u^{*} \in S$ and $i \in I$, for all $x \in S$, we have

$$
\begin{aligned}
& \frac{f_{i}(x)}{g_{i}(x)}-\frac{f_{i}\left(u^{*}\right)}{g_{i}\left(u^{*}\right)} \\
& =\frac{1}{g_{i}(x)}\left\{\left(x-u^{*}\right)^{T}\left[A_{i}-\frac{f_{i}\left(u^{*}\right)}{g_{i}\left(u^{*}\right)} B_{i}\right]\left(x-u^{*}\right)\right\} \\
& \quad+\frac{1}{g_{i}(x)}\left\{\left(\nabla f_{i}\left(u^{*}\right)-\frac{f_{i}\left(u^{*}\right)}{g_{i}\left(u^{*}\right)} \nabla g_{i}\left(u^{*}\right)\right)^{T}\left(x-u^{*}\right)\right\} .
\end{aligned}
$$

Therefore, for $\left(\tau^{*}, x^{*}\right) \in \mathscr{W} \times S$, we obtain

$$
\begin{aligned}
& \sum_{i=1}^{m} \tau_{i}^{*}\left(\frac{f_{i}\left(x^{*}\right)}{g_{i}\left(x^{*}\right)}-\frac{f_{i}\left(u^{*}\right)}{g_{i}\left(u^{*}\right)}\right) \\
& =\left(x^{*}-u^{*}\right)^{T}\left[F\left(\frac{\tau^{*}}{g\left(x^{*}\right)}, u^{*}\right)\right]\left(x^{*}-u^{*}\right) \\
& \quad+\sum_{i=1}^{m} \frac{\tau_{i}^{*}}{g_{i}\left(x^{*}\right)}\left(\nabla f_{i}\left(u^{*}\right)-\frac{f_{i}\left(u^{*}\right)}{g_{i}\left(u^{*}\right)} \nabla g_{i}\left(u^{*}\right)\right)^{T}\left(x^{*}-u^{*}\right),
\end{aligned}
$$

and since $F\left(\tau^{*} / g\left(x^{*}\right), u^{*}\right)$ is positive definite and $x^{*} \neq u^{*}$, then by (51)

$$
\begin{aligned}
0= & \sum_{i=1}^{m} \tau_{i}^{*}\left(\frac{f_{i}\left(x^{*}\right)}{g_{i}\left(x^{*}\right)}-\frac{f_{i}\left(u^{*}\right)}{g_{i}\left(u^{*}\right)}\right) \\
> & \sum_{i=1}^{m} \frac{\tau_{i}^{*}}{g_{i}\left(x^{*}\right)}\left(\nabla f_{i}\left(u^{*}\right)-\frac{f_{i}\left(u^{*}\right)}{g_{i}\left(u^{*}\right)} \nabla g_{i}\left(u^{*}\right)\right)^{T}\left(x^{*}-u^{*}\right) \\
\geqq & \min _{i \in I}\left\{\frac{1}{g_{i}\left(x^{*}\right)}\right\} \\
& \cdot \sum_{i=1}^{m} \tau_{i}^{*}\left(\nabla f_{i}\left(u^{*}\right)-\frac{f_{i}\left(u^{*}\right)}{g_{i}\left(u^{*}\right)} \nabla g_{i}\left(u^{*}\right)\right)^{T}\left(x^{*}-u^{*}\right) \geqq 0,
\end{aligned}
$$

which is a contradiction. 


\section{Conclusions}

The main contribution of this work is the development of Pareto optimality conditions for a particular vector optimization problem, where each objective function consists of a ratio of two quadratic functions with convexity being only assumed on the constraint set. We took advantage of the diagonalization of Hessian matrices. We have shown the relationship between the particular problem and two problems associated with it, and we use some assumptions of the linear combination of Hessian matrices to show the main duality theorems. For the particular problem, the results presented in this work might be useful to determine the termination criteria in the development of algorithms, and new extensions can be established to more general vector optimization problems, in which algorithms based on quadratic approximations are used locally. In future work we plan to develop algorithms using the concepts presented here.

\section{Conflict of Interests}

The authors declare that there is no conflict of interests regarding the publication of this paper.

\section{Acknowledgments}

The authors are indebted to the anonymous reviewers for their helpful comments. W. A. Oliveira was supported by Coordination for the Improvement of Higher Level Personnel of Brazil (CAPES). A. Beato-Moreno was partially supported by Spain's Ministry of Science and Technology under Grant MTM2007-63432. A. C. Moretti and L. L. Salles Neto were partially supported by National Council for Scientific and Technological Development of Brazil (CNPq) and Foundation for Research Support of the State of São Paulo (FAPESP).

\section{References}

[1] V. Chankong and Y. Y. Haimes, Multiobjective Decision Making: Theory and Methodology, vol. 8 of North-Holland Series in System Science and Engineering, North-Holland, Elsevier Science, New York, NY, USA, 1983.

[2] K. M. Miettinen, Nonlinear Multiobjective Optimization, Kluwer Academic Publishers, Boston, Mass, Usa, 1999.

[3] W. Dinkelbach, "On nonlinear fractional programming," Management Science, vol. 13, no. 7, pp. 492-498, 1967.

[4] R. Jagannathan, "Duality for nonlinear fractional programs," Zeitschrift für Operations Research, vol. 17, no. 1, pp. 1-3, 1973.

[5] T. Antczak, "Modified ratio objective approach in mathematical programming," Journal of Optimization Theory and Applications, vol. 126, no. 1, pp. 23-40, 2005.

[6] Z. A. Khan and M. A. Hanson, "On ratio invexity in mathematical programming," Journal of Mathematical Analysis and Applications, vol. 205, no. 2, pp. 330-336, 1997.

[7] L. V. Reddy and R. N. Mukherjee, "Some results on mathematical programming with generalized ratio invexity," Journal of Mathematical Analysis and Applications, vol. 240, no. 2, pp. 299310, 1999.
[8] V. Jeyakumar, "Strong and weak invexity in mathematical programming," Methods of Operations Research, vol. 55, pp. 109125, 1985.

[9] Z. A. Liang, H. X. Huang, and P. M. Pardalos, "Optimality conditions and duality for a class of nonlinear fractional programming problems," Journal of Optimization Theory and Applications, vol. 110, no. 3, pp. 611-619, 2001.

[10] B. D. Craven, Fractional Programming, vol. 4 of Sigma Series in Applied Mathematics, Heldermann, Berlin, Germany, 1988.

[11] T. Weir, "A note on invex functions and duality in generalized fractional programming," Research Report ACT 2600, Department of Mathematics, The University of New South Wales, New South Wales, Australia, 1990.

[12] Z. Liang, H. Huang, and P. M. Pardalos, "Efficiency conditions and duality for a class of multiobjective fractional programming problems," Journal of Global Optimization, vol. 27, no. 4, pp. 447-471, 2003.

[13] B. Mond and T. Weir, "Generalized concavity and duality," in Generalized Concavity Optimization and Economics, S. Schaible and W. T. Ziemba, Eds., pp. 263-280, Academic Press, New York, NY, USA, 1981.

[14] S. Schaible, "Duality in fractional programming: a unified approach," Operations Research, vol. 24, no. 3, pp. 452-461, 1976.

[15] C. R. Bector, "Duality in nonlinear fractional programming," Zeitschrift für Operations Research B, vol. 17, pp. A183-A193, 1973.

[16] C. R. Bector, S. Chandra, and C. Singh, "A linearization approach to multiobjective programming duality," Journal of Mathematical Analysis and Applications, vol. 175, no. 1, pp. 268279, 1993

[17] R. Osuna-Gómez, A. Rufián-Lizana, and P. Ruíz-Canales, "Multiobjective fractional programming with generalized convexity," Top, vol. 8, no. 1, pp. 97-110, 2000.

[18] L. B. Santos, R. Osuna-Gómez, and M. A. Rojas-Medar, "Nonsmooth multiobjective fractional programming with generalized convexity," Revista Integración, Escuela de Matemáticas, Universidad Industrial de Santander, vol. 26, no. 1, pp. 1-12, 2008.

[19] V. Jeyakumar and B. Mond, "On generalised convex mathematical programming," The Journal of the Australian Mathematical Society B: Applied Mathematics, vol. 34, no. 1, pp. 43-53, 1992.

[20] A. Cambini, J.-P. Crouzeix, and L. Martein, "On the pseudoconvexity of a quadratic fractional function," Optimization, vol. 51, no. 4, pp. 677-687, 2002.

[21] J. Crouzeix, J. A. Ferland, and S. Schaible, "Duality in generalized linear fractional programming," Mathematical Programming, vol. 27, no. 3, pp. 342-354, 1983.

[22] J. Y. Gotoh and H. Konno, "Maximization of the ratio of two convex quadratic functions over a polytope," Computational Optimization and Applications, vol. 20, no. 1, pp. 43-60, 2001.

[23] A. W. Lo and A. C. Mackinlay, "Maximizing predictability in the stock and bond markets," Macroeconomic Dynamics, vol. 1, no. 1, pp. 102-134, 1997.

[24] S. Schaible and J. Shi, "Recent developments in fractional programming: single-ratio and Max-Min case," in Proceedings of the International Conference on Nonlinear Analysis and Convex Analysis, pp. 493-506, 2003.

[25] H. P. Benson, "Fractional programming with convex quadratic forms and functions," European Journal of Operational Research, vol. 173, no. 2, pp. 351-369, 2006.

[26] M. T. Arévalo and A. Zapata, "Selección de carteras mediante programación fraccional multiobjetivo," in XXIII Congreso 
Nacional de Estadística e Investigación Operativa, Valencia, Spain, 1997.

[27] A. Beato, P. R. Canales, P. L. L. Calvo, and R. R. Bravo, "Multiobjective quadratic problem: characterization of the efficient points," in Generalized Convexity, Generalized Monotonicity: Recent Results, J. P. Crouzeix, J. E. Martinez-Legaz, and M. Volle, Eds., pp. 425-438, Kluwer Academic Publishers, Dordrecht, The Netherlands, 1998.

[28] A. Beato, R. I. Macias, and P. R. Canales, "Decisión multicriterio a través de problemas de optimizacion cuadrático-fraccionales," The Revista de la Real Academia de Ciencias Exactas, Físicas y Naturales, España, vol. 93, no. 4, pp. 499-505, 1999.

[29] H. Konno and M. Inori, "Bond portfolio optimization by bilinear fractional programming," Journal of the Operations Research Society of Japan, vol. 32, no. 2, pp. 143-158, 1989.

[30] P. Korhonen and G. Y. Yu, "A reference direction approach to multiple objective quadratic-linear programming," European Journal of Operational Research, vol. 102, no. 3, pp. 601-610, 1997.

[31] P. Korhonen and G. Y. Yu, "On computing objective function values in multiple objective quadratic-linear programming," European Journal of Operational Research, vol. 106, no. 1, pp. 184-190, 1998.

[32] J. S. H. Kornbluth and R. E. Steuer, "Multiple objective linear fractional programming," Management Science, vol. 27, no. 9, pp. 1024-1039, 1981.

[33] R. Rhode and R. Weber, "Multiple objective quadratic-linear programming," in Operational Research, North-Holland Publishing, Amsterdam, The Netherlands, J. P. Brans edition, 1981.

[34] V. Pareto, Cours d'Economie Politique, Rouge, Lausanne, Switzerland, 1896.

[35] T. Maeda, "Constraint qualifications in multiobjective optimization problems: differentiable case," Journal of Optimization Theory and Applications, vol. 80, no. 3, pp. 483-500, 1994.

[36] M. Guignard, "Generalized Kuhn-Tucker conditions for mathematical programming problems in a Banach space," SIAM Journal on Control, vol. 7, pp. 232-241, 1969.

[37] A. M. Geoffrion, "Proper efficiency and the theory of vector maximization," Journal of Mathematical Analysis and Applications, vol. 22, pp. 618-630, 1968.

[38] P. Wolfe, "A duality theorem for non-linear programming," Quarterly of Applied Mathematics, vol. 19, pp. 239-244, 1961. 


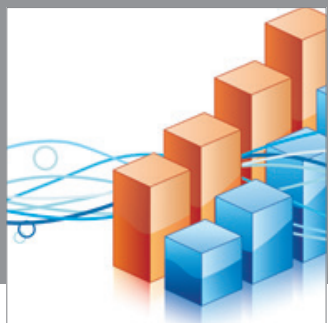

Advances in

Operations Research

mansans

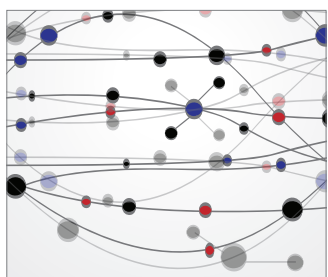

The Scientific World Journal
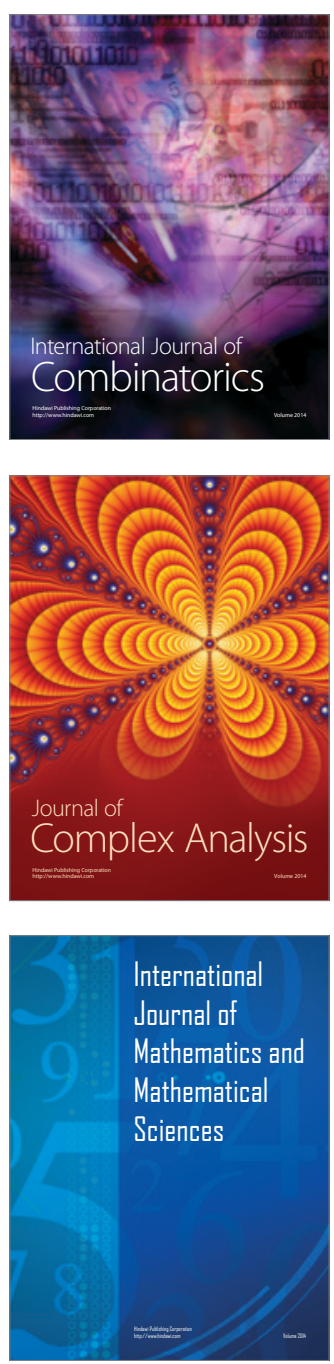
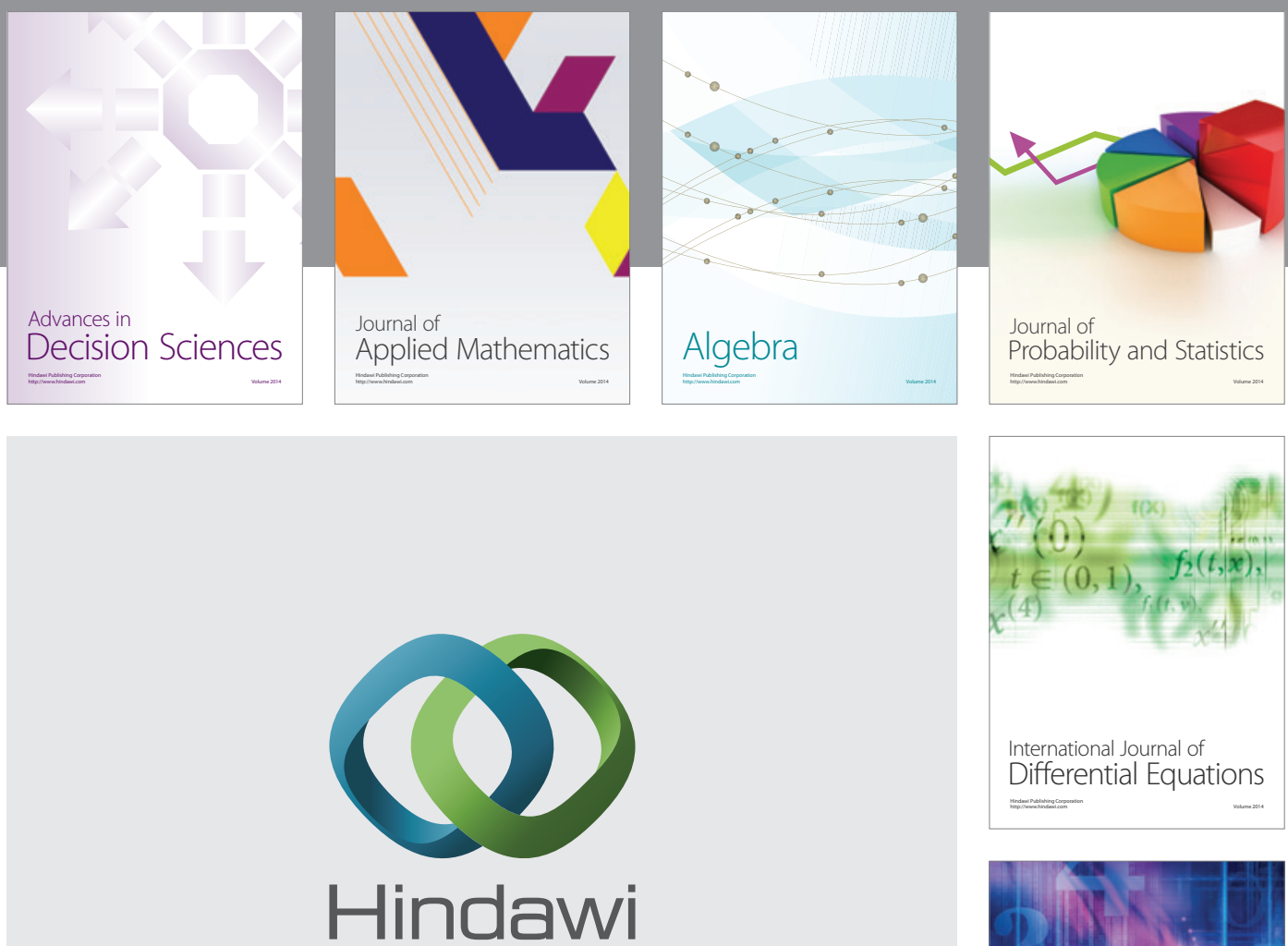

Submit your manuscripts at http://www.hindawi.com
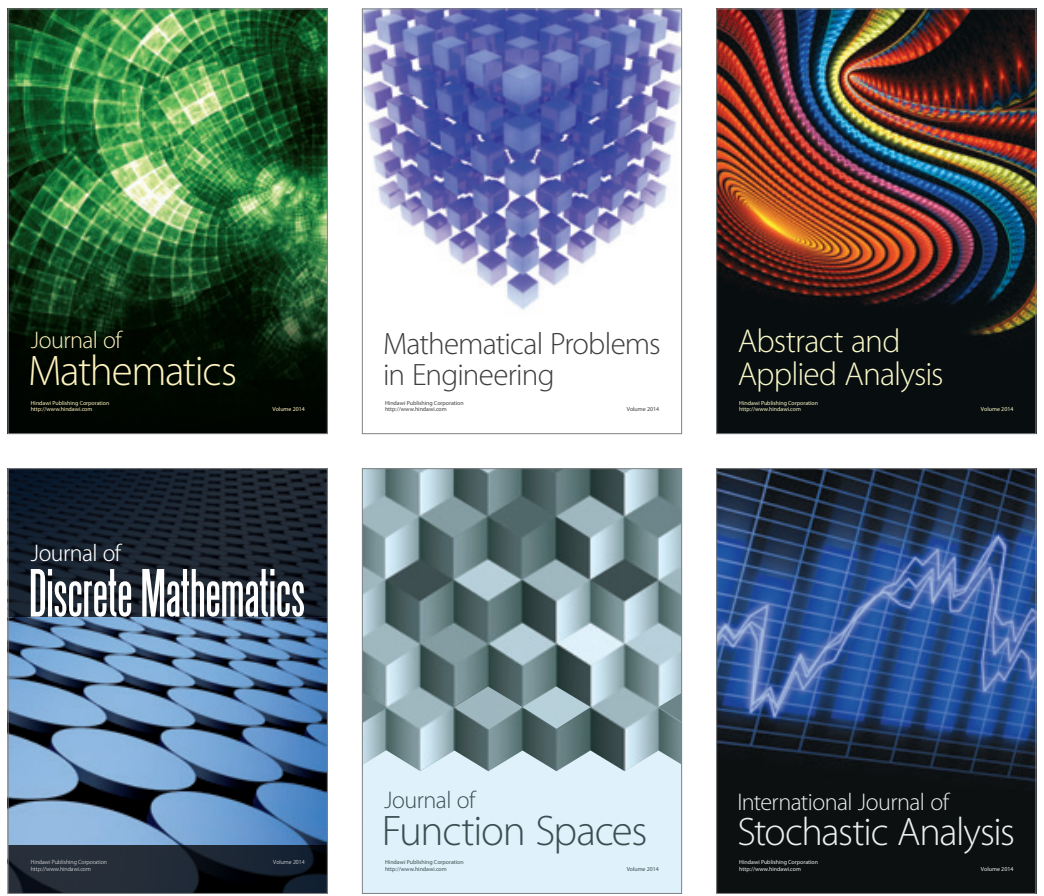

Journal of

Function Spaces

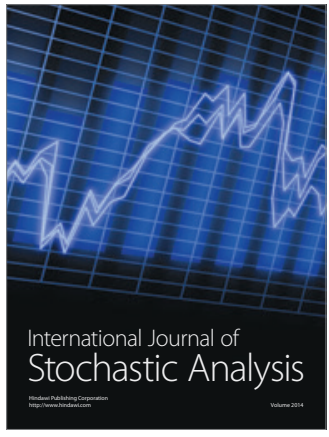

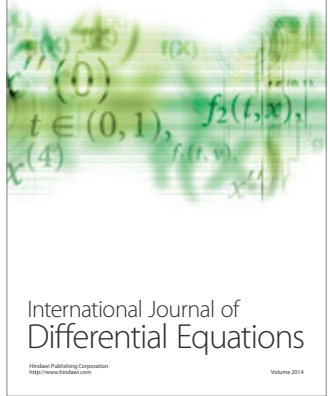
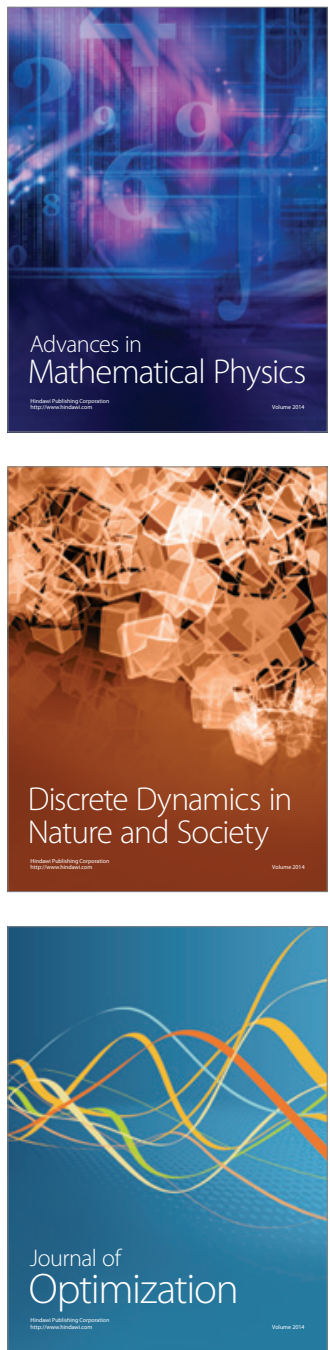